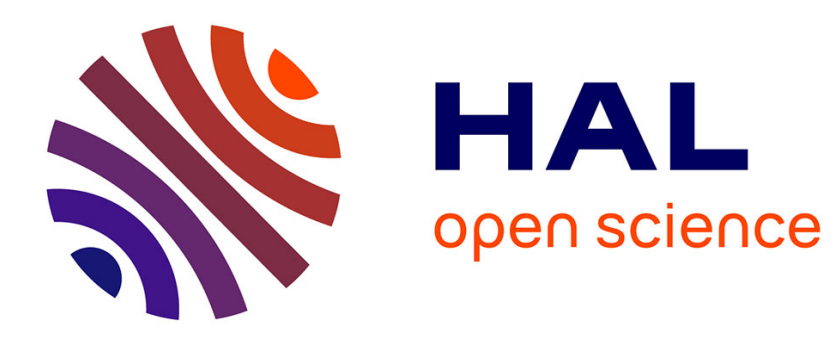

\title{
Homogenization of an elastic media with gaseous inclusions
}

\author{
Léonardo Baffico, Céline Grandmont, Yvon Maday, Axel Osses
}

\section{To cite this version:}

Léonardo Baffico, Céline Grandmont, Yvon Maday, Axel Osses. Homogenization of an elastic media with gaseous inclusions. Multiscale Modeling and Simulation: A SIAM Interdisciplinary Journal, 2008, 7 (1), pp.33. inria-00180307

\section{HAL Id: inria-00180307 https://hal.inria.fr/inria-00180307}

Submitted on 18 Oct 2007

HAL is a multi-disciplinary open access archive for the deposit and dissemination of scientific research documents, whether they are published or not. The documents may come from teaching and research institutions in France or abroad, or from public or private research centers.
L'archive ouverte pluridisciplinaire HAL, est destinée au dépôt et à la diffusion de documents scientifiques de niveau recherche, publiés ou non, émanant des établissements d'enseignement et de recherche français ou étrangers, des laboratoires publics ou privés. 


\title{
Homogenization of an elastic media with gaseous inclusions
}

\author{
L. Baffico* \\ C. Grandmont ${ }^{\dagger}$ \\ Y. Maday \\ A. Osses $\S$
}

October 10, 2007

\begin{abstract}
We study the asymptotic behavior of a system modeling a composite material made of an elastic periodically perforated support, with period $\varepsilon>0$, and a perfect gas placed in each of these perforations, as $\varepsilon$ goes to zero. The model we use is linear corresponding to deformations around a reference configuration. We apply both two-scale asymptotic expansion and two-scale convergence methods in order to identify the limit behaviors as $\varepsilon$ goes to 0 . We state that in the limit, we get a two-scale linear elasticity-like boundary value problem. From this problem, we identify the corresponding homogenized and periodic cell equations which allows us to find the first corrector term. The analysis is performed both in the case of an incompressible and compressible material. We derive some mechanical properties of the limit materials by studying the homogenized coefficients. Finally, we numerically calculate the homogenized coefficients in the incompressible case, for different types of elastic materials.
\end{abstract}

AMS subject classification: 93A90, 35B27, 35B40, 74F10

Key words: Mathematical modeling; Periodic homogenization; Asymptotic analysis; Fluidstructure interaction.

\footnotetext{
*Université de Caen, UMR 6139, Laboratoire de Mathématiques N. Oresme, BP 5186 F-14032 Caen, France

${ }^{\dagger}$ INRIA, Projet REO, Rocquencourt, BP 105 F-78153 Le Chesnay Cedex, France

${ }^{\ddagger}$ Université Pierre et Marie Curie-Paris6, UMR 7598, Laboratoire J.-L. Lions, Paris, F-75005 France and Division of Applied Mathematics, Brown University 182 George Street, Providence, RI 02912, USA.

${ }^{\S}$ DIM-FCFM, U. de Chile, Santiago, Chile \& CMM UMI 2807 CNRS-UChile, Santiago, Chile. This work has been partially supported by FONDECYT 1061263, ECOS-CONICYT CO4E08 grants and ACI project "le-poumonvous-dis-je"
} 


\section{Introduction}

\subsection{Preface}

In the last decades, a growing interest in the use of both mathematical and numerical tools to study human physiological phenomena has been observed, e.g. in the study of the blood circulation system, respiratory system, heart's electrical activity, brain deformation, etc..

Our motivation concerns the mathematical modeling of the respiratory system and this paper belongs to a series relative to this topic $([1],[2],[26],[17],[18])$. Our interest here is to provide the basics to get a model for the behavior of the alveolar region. The coarse description of the respiratory system on which this analysis enters can be described as follows (see for example, [19], [36], [37] for more details): we assume that the respiratory system can be separated into three distinct parts. First, the upper part composed of the mouth and nose, larynx and pharynx, secondly, the trachea and bronchial tree (subdivided in proximal part, from $1^{\text {st }}$ to $5^{\text {th }}-6^{\text {th }}$ generation, and distal part, from $6^{t h}-7^{\text {th }}$ to $13^{\text {th }}$ generation), lastly, the lungs composed by the set of terminal acini (which are, in its turn, composed of the terminal bronchioles and the alveoli), the whole being imbedded in a structural matrix, called the parenchyma.

It is quite an easy matter to realize that the exact representation of a flow governed by the Navier-Stokes equations, in this complex 3D geometry, is far from being achievable currently. This will only be feasible, though still quite expensive, for the upper airways and the bronchial proximal part ([15], [25]). For the other parts, we need to perform some model reduction. For the bronchial distal part, we may refer to a reduction technique, as the one advocated in the blood circulation [30] and in the bronchial tree [27], or to a more sophisticated approach [24]. Concerning the terminal part, the description of the set of acini may be obtained thanks to the homogenization framework, either fractal homogenization as in [7], or periodic homogenization for the alveola part, involving fluid structure interaction, which is the subject of the present paper.

This paper is a first step in this direction as it is based on serious simplifications of the parenchyma-alveolar system. First of all, we restrain our study to the stationary case, and we assume that the number of air molecules in each alveolus remains constant (e.g., more or less, the case of an excised lung filled with air, at a given pressure, and then sealed in order to analyze its mechanical behavior [35]). Next, we assume that air behaves like a perfect compressible gas, and that parenchyma behaves like a linear elastic material (the deformations are small). Finally, the space repetition of the acini suggests us to consider a periodically perforated elastic material with period $\epsilon>0$. Since in our framework each alveolus is isolated, we will not consider the terminal bronchioles as part of the geometry.

The material under study here is rather like a foam, for which we did not find any analysis in the literature through homogenization. We first study the case of a compressible elastic material and then, the incompressible case (which is a good assumption for the parenchyma). In the first case, the behavior of the elastic deformation field is governed by the linear elasticity system (see [8], [31]) and the physical properties of the elastic material are determined by the Lamé constants $\mu$ and $\lambda$ (or, equivalently, by the Young modulus $E$ and Poisson ratio $\nu$ ). The incompressible case can be obtained from the first model by letting $\lambda$ go to infinity, and then, the elastic deformation field is governed by a Stokes-like system (in this case $\nu=1 / 2$ ). In both cases, the interaction of the elastic material and the gas leads, after linearization, to a non-standard boundary condition on the bubble's walls. The boundary condition is of non-local type, similar to the case considered in other fluid-structure problems [10], [12]. Then, we obtain boundary value problems (13) and (14), for the compressible and incompressible case, respectively. In the incompressible case, when the effect of gas pressure is neglected, the well-known homogenization of the Stokes-perforated 
domain model is obtained (see [32], [33]).

Once the geometry and models are set up, we first study the well-posedness of both problems and then, using some homogenization techniques; we analyze the asymptotic behavior of the displacement field solutions of (13) and (14) as $\epsilon$ goes to 0 . The main results of this paper are the two-scale convergence theorems 2.4 and 3.5, for the compressible and incompressible models, respectively. From these results it is possible to identify the macroscopic homogenized problems (see (63)-(64) and (85)-(87), respectively). To study the well-posedness of the homogenized problem, we use both the method based on a Korn-type inequality in the two-scale limit problem [3] and the method of direct existence and uniqueness of the final homogenized problem after eliminating the microscopic variable [10].

A physical interpretation of the homogenized problem can be performed: In the case of a compressible elastic material, the macroscopic effect of the gaseous bubbles can be observed as a modification, as expected, of the compressibility (the second Lamé constant $\lambda$ ) of the limit material (see (64)). In the incompressible case, these effects are more noticeable since a new macroscopic compressibility-like constant appears in the limit problem (see (85)- (87)). This new constant only depends in microscopic characteristics of the bubble (gas pressure and geometry). We can observe that in the case of a simple perforated incompressible media (i.e., the case of void inclusions) this constant is equal to 0 . Therefore the added compressibility of the limit material is mainly due to the presence of the perfect gas inside each bubble.

Finally, we calculate the homogenized coefficients in the incompressible case, for different geometries and elastic materials (determined by the first Lamé constant $\mu$ ). We compare the case of void inclusions with the case of a gas at atmospheric pressure. The results show that in the case of a soft elastic material (such as living tissues), the absence or presence of gas in the bubbles produces significant variations in the homogenized coefficients. On the other hand, if a stiffer material is considered (such as rubber), a slighter influence of the bubbles' gas on the homogenized coefficients can be observed. The main effect here is for incompressible materials that become compressible.

One may quote other works related to lung modeling such as [29], where the alveoli structure is considered as a porous media and a formal homogenization is performed on the linearized Navier-Stokes equations coupled to linear viscoelasticity. Another related article is [23], where a computational algorithm is proposed in order to generate the acinus geometry. In [20] the propagation of elastic waves in the thorax is studied. The lung, that is supposed to be homogeneous, belongs to a stratified domain that represents the thoracic cage.

Concerning homogenization theory for related fluid-structure interaction problems one may refer to [14] where the homogenization of a thin elastic structure immersed in a viscous fluid is studied. In this work both fluid and solid domains are connected subsets. In [9] the homogenization of a perforated media with time-oscillating perforation is studied.

In our case, we consider non connected alveoli. This first approximation gives an idea of the elastic properties of the media, that could be validated in some cases, as when considering impact forces [21]. Indeed, under impact forces, the characteristic time of deformations is small compared with the characteristic time of air-transport between alveoli. In any case, a more complete model should be considered by coupling the homogenized model presented here with the respiratory tree represented as a dissipative term in the equations following the ideas of [17].

In a forthcoming analysis we can use the reduced model obtained by the homogenization process in a geometry acquired from medical images and couple this system to a network of pipes, representing either a real or a artificial representing the upper and proximal parts introduced above. The coupling could be at the level of the boundary conditions in the network where the flow rate would be computed from the volume variations of the parenchyma. 


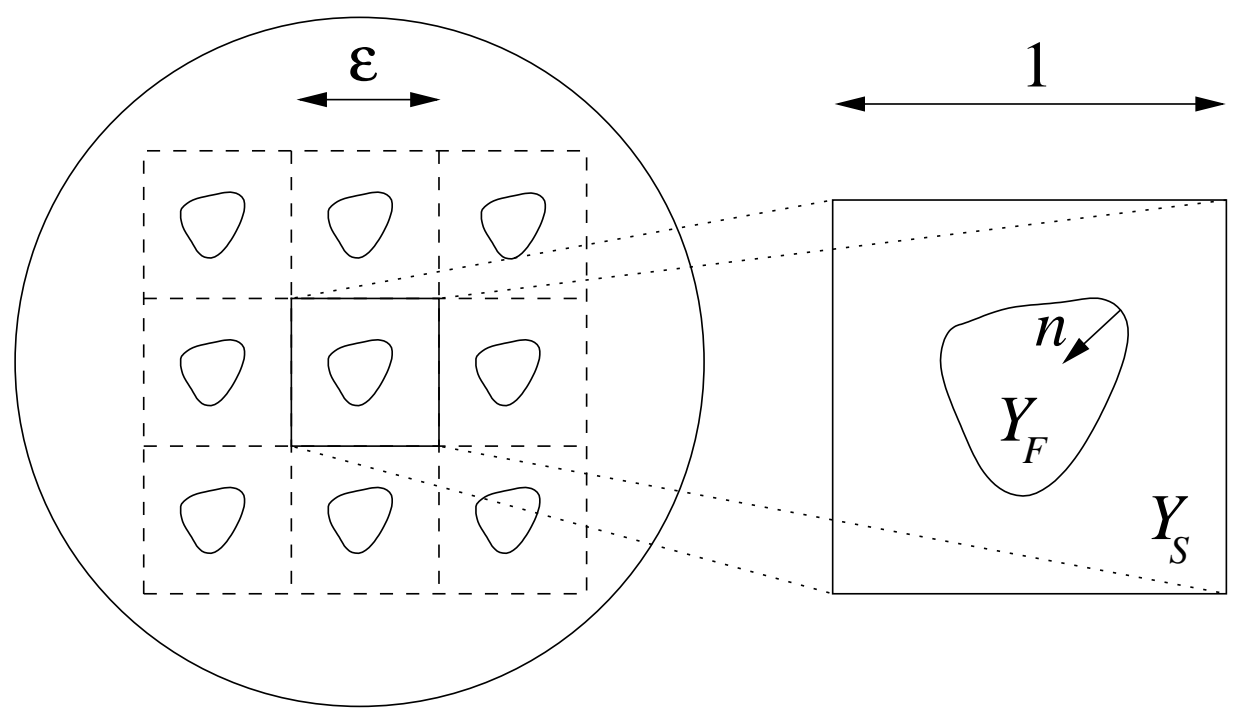

Figure 1: The domain $\Omega^{\varepsilon}$ and the fluid-structure unit cell $Y$.

The paper is organized as follows. In the remaining part of section 1 we present the geometry of the perforated elastic media, and then we describe the models that we shall study. In section 2 , we formally derive the homogenized limit of the compressible model (13) by a two-scale asymptotic expansion and then we justify it in the two-scale convergence framework. Then, in section 3, we carry out the same analysis for the incompressible model (14). Finally, in section 4, through numerical simulations, we compute the homogenized coefficients to test the homogenized model with parameters inspired in realistic lung's anatomic data.

\subsection{Geometry}

Let $\Omega$ be a regular nonempty bounded open subset in $\mathbb{R}^{N}(N=2$ or 3$)$ with boundary $\Gamma$ and unit outward normal $n$. The boundary is decomposed as $\bar{\Gamma}=\bar{\Gamma}_{D} \cup \bar{\Gamma}_{N}$, where $\Gamma_{D}$ and $\Gamma_{N}$ are such that $\Gamma_{D} \cap \Gamma_{N}=\emptyset$ and $\left|\Gamma_{D}\right|>0$; they support, respectively, Dirichlet and Neumann boundary conditions.

Let $Y=(-1 / 2,1 / 2)^{N}$ be the unit cell decomposed into two nonempty open sets: $Y_{F}$ that represents a simply connected bubble and $Y_{S}$ that represents the elastic support. They are such that

$$
\bar{Y}=\bar{Y}_{S} \cup \bar{Y}_{F}, \quad Y_{S} \cap Y_{F}=\emptyset, \quad \bar{Y}_{F} \subset Y .
$$

For instance, $Y_{F}$ could be an open ball $B(0, r)$ centered at 0 with radius $r<1 / 2$. Given a small parameter $\varepsilon>0$ and a multi index $k=\left(k_{1}, \ldots, k_{N}\right) \in \mathbb{Z}^{N}$, we define

$$
Y^{\varepsilon, k}=\varepsilon(Y+k), \quad Y_{F}^{\varepsilon, k}=\varepsilon\left(Y_{F}+k\right), \quad Y_{S}^{\varepsilon, k}=\varepsilon\left(Y_{S}+k\right), \quad \Gamma^{\varepsilon, k}=\partial Y_{F}^{\varepsilon, k},
$$

that is, a translation and a homothetic transformation of the reference cells $Y, Y_{S}, Y_{F}$ and of its interface. By introducing the multi index set

$$
\mathbb{Z}_{\varepsilon}^{N}=\left\{k \in \mathbb{Z}^{N}: \overline{Y^{\varepsilon, k}} \subset \Omega\right\},
$$

we define the periodically perforated domain and its interior boundary as

$$
\Omega^{\varepsilon}=\Omega \backslash \bigcup_{k \in \mathbb{Z}_{\varepsilon}^{N}} \overline{Y_{F}^{\varepsilon, k}}, \quad \Gamma^{\varepsilon}=\bigcup_{k \in \mathbb{Z}_{\varepsilon}^{N}} \Gamma^{\varepsilon, k}
$$


with unit outward normal $n^{\varepsilon}$ (note that, with this definition, $n^{\varepsilon}$ points out into the interior of $Y_{F}^{\varepsilon, k}$, see figure 1) that we will simply denote by $n$ if there is no possible confusion.

Let $\chi^{\varepsilon, k}, \chi_{F}^{\varepsilon, k}$ and $\chi_{S}^{\varepsilon, k}$ be the characteristic functions of $Y^{\varepsilon, k}, Y_{F}^{\varepsilon, k}$ and $Y_{S}^{\varepsilon, k}$ in $L^{2}(\Omega)$, respectively, and $\chi_{F}^{\varepsilon}$ and $\chi_{S}^{\varepsilon}$ the characteristic functions of $\bigcup_{k \in \mathbb{Z}_{\varepsilon}^{N}} Y_{F}^{\varepsilon, k}$ and $\Omega^{\varepsilon}$, respectively. Note that we have the following relations

$$
\chi_{F}^{\varepsilon}=\sum_{k \in \mathbb{Z}_{\varepsilon}^{N}} \chi_{F}^{\varepsilon, k}, \quad \chi_{S}^{\varepsilon}=1-\chi_{F}^{\varepsilon} .
$$

\subsection{Description of the models}

We suppose that $\Omega^{\varepsilon}$ is the reference configuration for the elastic media for a given constant external pressure $p_{a}>0$. The external pressure $p_{a}$ is also the reference pressure into the inclusions that we consider impermeable, so the number $N_{\varepsilon}$ of gas moles into each bubble at a given constant temperature $T_{a}>0$ is given by the law of ideal gases:

$$
N_{\varepsilon} R T_{a}=\varepsilon^{N}\left|Y_{F}\right| p_{a},
$$

where $\varepsilon^{N}\left|Y_{F}\right|$ is the prescribed volume of each bubble in the reference configuration and $R=$ $k_{B} N_{A}$, where $N_{A}>0$ is the Avogadro's number and $k_{B}>0$ is the Boltzmann constant. We are interested in the small deformations of the elastic media around the reference state, and thus to the variation of the displacement associated with the variations of the bubbles pressure.

We shall analyze two cases: firstly, we will consider a linear homogeneous elastic material and secondly, we will add to this model an incompressibility constraint.

Since we assume that the gas is perfect, the pressure $p^{\varepsilon, k}$ and the volume $V^{\varepsilon, k}$ of the deformed $k$-bubble satisfy the law

$$
p^{\varepsilon, k}=\frac{N_{\varepsilon} R T_{a}}{V^{\varepsilon, k}}=\frac{\varepsilon^{N}\left|Y_{F}\right|}{V^{\varepsilon, k}} p_{a},
$$

where we have used (6). If we denote by $d^{\varepsilon}$ the displacement field of the elastic media, then the volume can be expressed in term of $d^{\varepsilon}$ or of the deformation map $\varphi_{\varepsilon}(x)=x+d^{\varepsilon}(x)$ as

$$
V^{\varepsilon, k}=G_{\varepsilon, k}\left(d^{\varepsilon}\right)=\int_{Y_{F}^{\varepsilon, k}} \operatorname{det} \nabla \varphi_{\varepsilon}(x) d x
$$

where $d^{\varepsilon}$ has been extended to the interior of the inclusions in a way that will be specified later.

This volume can be written thanks to an integration by parts (see [8] p. 83 and remind that the normal $n^{\varepsilon}$ points out into $\left.Y_{F}^{\varepsilon, k}\right)$ in the following way:

$$
G_{\varepsilon, k}\left(d^{\varepsilon}\right)=-\frac{1}{N} \int_{\Gamma^{\varepsilon, k}} \varphi_{\varepsilon}(x) \cdot \operatorname{Cof} \nabla \varphi_{\varepsilon}(x) n^{\varepsilon} d \gamma .
$$

Here $\operatorname{Cof} A=(\operatorname{det} A) A^{-t}$ denotes the cofactor matrix. The idea is now to linearize (9). The Gâteaux derivative of the functional $G_{\varepsilon, k}$ is given by (see [8] p. 83)

$$
G_{\varepsilon, k}^{\prime}(b) \theta=-\frac{1}{N} \int_{\Gamma^{\varepsilon, k}} \theta \cdot \operatorname{Cof} \nabla(x+b(x)) n^{\varepsilon} d \gamma .
$$

Consequently, we can approximate the deformed volume of the $k$-bubble by

$$
V^{\varepsilon, k} \approx G_{\varepsilon, k}(0)+G_{\varepsilon, k}^{\prime}(0) d^{\varepsilon}=\varepsilon^{N}\left|Y_{F}\right|-\frac{1}{N} \int_{\Gamma^{\varepsilon, k}} d^{\varepsilon} \cdot n^{\varepsilon} d \gamma
$$


Then the corresponding pressure given by (7) can be approximated by

$$
p_{\varepsilon}^{k}=\frac{\varepsilon^{N}\left|Y_{F}\right|}{V^{\varepsilon, k}} p_{a} \approx\left(1+\frac{1}{N \varepsilon^{N}\left|Y_{F}\right|} \int_{\Gamma^{\varepsilon, k}} d^{\varepsilon} \cdot n^{\varepsilon} d \gamma\right) p_{a}
$$

Hence, at first order, the variation of the pressure inside the $k$-bubble depends on the average of the normal displacement on the interface and is given by

$$
p_{\varepsilon}^{k}-p_{a} \approx \frac{A}{\varepsilon^{N}}\left(\int_{\Gamma^{\varepsilon, k}} d^{\varepsilon} \cdot n^{\varepsilon} d \gamma\right), \quad \text { with } A=\frac{p_{a}}{N\left|Y_{F}\right|},
$$

where we have introduced the constant $A$ that we will use through the whole paper. Therefore, in the first case that we shall analyze, the displacement field $d^{\varepsilon}$ satisfies the linearized elasticity equations with non standard boundary conditions on the fluid-solid interface:

$$
\left\{\begin{aligned}
-\operatorname{div} \sigma\left(d^{\varepsilon}\right) & =f_{S}, & & \text { in } \Omega^{\varepsilon}, \\
\sigma\left(d^{\varepsilon}\right) n^{\varepsilon}+\frac{A}{\varepsilon^{N}}\left(\int_{\Gamma^{\varepsilon, k}} d^{\varepsilon} \cdot n^{\varepsilon} d \gamma\right) n^{\varepsilon} & =0, & & \text { on } \Gamma^{\varepsilon, k}, \forall k \in \mathbb{Z}_{\varepsilon}^{N}, \\
\sigma\left(d^{\varepsilon}\right) n & =0, & & \text { on } \Gamma_{N}, \\
d^{\varepsilon} & =0, & & \text { on } \Gamma_{D},
\end{aligned}\right.
$$

where $\sigma\left(d^{\varepsilon}\right)$ stands for the stress tensor associated with $d^{\varepsilon}$, i.e.

$$
\sigma\left(d^{\varepsilon}\right)=\lambda \operatorname{div} d^{\varepsilon} I+2 \mu e\left(d^{\varepsilon}\right),
$$

with $\lambda>0$ and $\mu>0$, the Lamé constants of the elastic media, and $e\left(d^{\varepsilon}\right)=1 / 2\left(\nabla d^{\varepsilon}+\left(\nabla d^{\varepsilon}\right)^{T}\right)$ stands for the strain tensor. Finally, $f_{S}$ represents an external force field acting in the media (e.g. gravity force).

Note that this type of non-local boundary conditions appears in other fluid-structure models, for instance, when studying vibrations of rigid tubes immersed in a fluid (see [5], [12]).

It is possible to consider a non-homogeneous Neumann boundary condition on $\Gamma_{N}$ that would represent a pressure force acting on this boundary.

In the second case that we shall study, we consider a linear incompressible media corresponding to the following model:

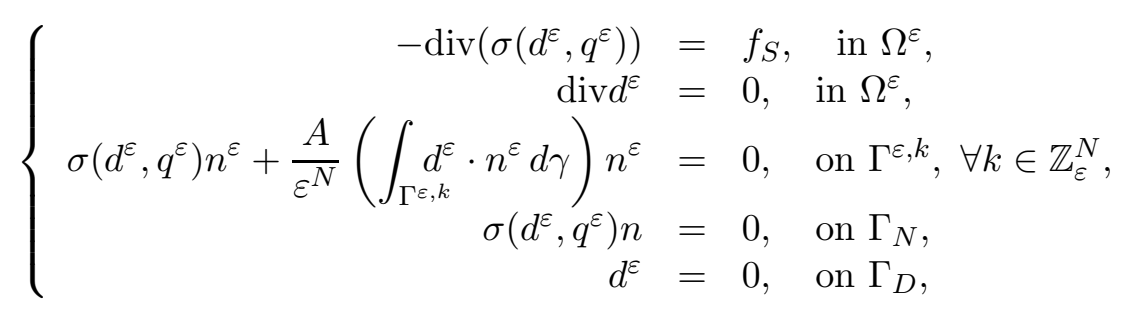

where now the stress tensor $\sigma\left(d^{\varepsilon}, q^{\varepsilon}\right)$ is defined by

$$
\sigma\left(d^{\varepsilon}, q^{\varepsilon}\right)=2 \mu e\left(d^{\varepsilon}\right)-q^{\varepsilon} I .
$$

The constraint $\operatorname{div} d^{\varepsilon}=0$ corresponds to the linearized version of $\operatorname{det}\left(\nabla \varphi^{\varepsilon}\right)=1$, which states that the body is incompressible, and $q^{\varepsilon}$ is the Lagrange multiplier associated with this constraint. Note that (14) can be obtained from (13) by letting $\lambda$ tend to $+\infty$ (see section 3.2, remark 3.5). 


\section{Compressible case: homogenization process}

The analysis will be divided into several steps: first we prove that (13) is well-posed and we derive $a$ priori estimates independent of the parameter $\varepsilon$. Afterwards the homogenized problem is obtained formally thanks to a two scale asymptotic expansion and justified by the two scale convergence method. Finally the limit problem is studied: in particular we exhibit the homogenized stress tensor and prove that the limit problem is well-posed.

\subsection{Variational formulation and a priori estimates}

In this section we present the variational formulation of problem (13) and we derive an existence and uniqueness result for it. Afterward, using the variational formulation, we obtain a priori estimates independent of $\varepsilon$ on the solutions of (13).

\subsubsection{Variational formulation}

Let $X_{D}^{\varepsilon}=\left\{v \in H^{1}\left(\Omega^{\varepsilon}\right)^{N}|v|_{\Gamma_{D}}=0\right\}$ and $X_{D}=\left\{v \in H^{1}(\Omega)^{N}|v|_{\Gamma_{D}}=0\right\}$ where $\left.v\right|_{\Gamma_{D}}$ denotes the trace of $v$ on $\Gamma_{D}$. Let us denote by $\|\cdot\|_{0, \Omega^{\varepsilon}}$ and $\|\cdot\|_{0, \Omega}$ their respective $L^{2}$-norms and by $|\cdot|_{1, \Omega^{\varepsilon}}=\|\nabla \cdot\|_{0, \Omega^{\varepsilon}}$ and $|\cdot|_{1, \Omega}=\|\nabla \cdot\|_{0, \Omega}$ their respective $H^{1}$ Sobolev seminorms.

It is easy to see that the variational formulation of (13) is

$$
\left\{\begin{array}{l}
\text { Find } d^{\varepsilon} \in X_{D}^{\varepsilon} \text { such that } \\
a^{\varepsilon}\left(d^{\varepsilon}, \varphi^{\varepsilon}\right)=\ell^{\varepsilon}\left(\varphi^{\varepsilon}\right), \quad \forall \varphi^{\varepsilon} \in X_{D}^{\varepsilon},
\end{array}\right.
$$

where $a^{\varepsilon}(\cdot, \cdot): X_{D}^{\varepsilon} \times X_{D}^{\varepsilon} \rightarrow \mathbb{R}$ is the bilinear form defined by

$$
a^{\varepsilon}\left(d^{\varepsilon}, \varphi^{\varepsilon}\right)=a_{\mu}^{\varepsilon}\left(d^{\varepsilon}, \varphi^{\varepsilon}\right)+a_{\lambda}^{\varepsilon}\left(d^{\varepsilon}, \varphi^{\varepsilon}\right)+a_{\Gamma}^{\varepsilon}\left(d^{\varepsilon}, \varphi^{\varepsilon}\right),
$$

with

$$
\begin{aligned}
& a_{\mu}^{\varepsilon}\left(d^{\varepsilon}, \varphi^{\varepsilon}\right)=2 \mu \int_{\Omega^{\varepsilon}} e\left(d^{\varepsilon}\right): e\left(\varphi^{\varepsilon}\right) d x, \\
& a_{\lambda}^{\varepsilon}\left(d^{\varepsilon}, \varphi^{\varepsilon}\right)=\lambda \int_{\Omega^{\varepsilon}} \operatorname{div} d^{\varepsilon} \operatorname{div} \varphi^{\varepsilon} d x, \\
& a_{\Gamma}^{\varepsilon}\left(d^{\varepsilon}, \varphi^{\varepsilon}\right)=\frac{A}{\varepsilon^{N}} \sum_{k \in \mathbb{Z}_{\varepsilon}^{N}}\left(\int_{\Gamma^{\varepsilon, k}} d^{\varepsilon} \cdot n d \gamma\right)\left(\int_{\Gamma^{\varepsilon, k}} \varphi^{\varepsilon} \cdot n d \gamma\right),
\end{aligned}
$$

and $\ell^{\varepsilon}(\cdot): X_{D}^{\varepsilon} \rightarrow \mathbb{R}$ is the linear form defined by

$$
\ell^{\varepsilon}\left(\varphi^{\varepsilon}\right)=\int_{\Omega^{\varepsilon}} f_{S} \cdot \varphi^{\varepsilon} d x, \text { with } f_{S} \in L^{2}(\Omega) .
$$

It is well known that Poincaré's and Korn's inequalities hold in $X_{D}^{\varepsilon}$, that is, there exist $\alpha_{\varepsilon}>0$ and $\gamma_{\varepsilon}>0$, constants only depending on $\Omega^{\varepsilon}$, such that (see [13])

$$
\|\left. d^{\varepsilon}\right|_{0, \Omega^{\varepsilon}} \leq \gamma_{\varepsilon}\left|d^{\varepsilon}\right|_{1, \Omega^{\varepsilon}}, \quad \forall d^{\varepsilon} \in X_{D}^{\varepsilon},
$$

and

$$
\|\left. e\left(d^{\varepsilon}\right)\right|_{0, \Omega^{\varepsilon}} \geq \alpha_{\varepsilon}\left|d^{\varepsilon}\right|_{1, \Omega^{\varepsilon}}, \quad \forall d^{\varepsilon} \in X_{D}^{\varepsilon} .
$$

Therefore, since $\mu, \lambda>0$ and $A>0$ (note that this implies that $a_{\Gamma}^{\varepsilon}\left(d^{\varepsilon}, d^{\varepsilon}\right)$ is positive), for each $\varepsilon>0$, the bilinear form $a^{\varepsilon}$ is continuous and coercive. Hence by Lax-Milgram lemma, there exists a unique solution to (15). 


\subsubsection{A priori estimates}

Since $\alpha_{\varepsilon}$ and $\gamma_{\varepsilon}$ depend on $\varepsilon$, the estimates that we could derive will not be uniform on $\varepsilon$. However, it is known (see for instance [9] or the appendix in [10]) that there exists an extension operator $\sim: X_{D}^{\varepsilon} \rightarrow X_{D}$ such that

$$
\widetilde{\varphi^{\varepsilon}}=\varphi^{\varepsilon} \text { in } \Omega^{\varepsilon}, \quad\left\|e\left(\widetilde{\varphi^{\varepsilon}}\right)\right\|_{0, \Omega} \leq K\left\|e\left(\varphi^{\varepsilon}\right)\right\|_{0, \Omega^{\varepsilon}}, \quad \forall \varphi^{\varepsilon} \in X_{D}^{\varepsilon},
$$

where $K>0$ does not depend on $\varepsilon$. Since Poincaré's and Korn's inequalities also hold in $X_{D}$, it follows that for all $\varphi^{\varepsilon} \in X_{D}^{\varepsilon}$ :

$$
\left\|e\left(\varphi^{\varepsilon}\right)\right\|_{0, \Omega^{\varepsilon}} \geq \frac{1}{K} \|\left. e\left(\widetilde{\varphi^{\varepsilon}}\right)\right|_{0, \Omega} \geq \frac{\alpha_{0}}{K}\left|\widetilde{\varphi^{\varepsilon}}\right|_{1, \Omega} \geq \frac{\alpha_{0}}{K}\left|\varphi^{\varepsilon}\right|_{1, \Omega^{\varepsilon}}
$$

and

$$
\left\|\left.\varphi^{\varepsilon}\right|_{0, \Omega^{\varepsilon}} \leq\right\| \widetilde{\varphi^{\varepsilon}}\left\|_{0, \Omega} \leq \gamma_{0}\left|\widetilde{\varphi^{\varepsilon}}\right|_{1, \Omega} \leq \frac{K}{\alpha_{0}} \gamma_{0}\right\| e\left(\varphi^{\varepsilon}\right) \|_{0, \Omega^{\varepsilon}} \leq \frac{K \gamma_{0}}{\alpha_{0}}\left|\varphi^{\varepsilon}\right|_{1, \Omega^{\varepsilon}}
$$

with $\alpha_{0}>0$ and $\gamma_{0}>0$ depending only on $\Omega$. This shows in fact that $|\cdot|_{1, \Omega^{\varepsilon}},\left.|| e(\cdot)\right|_{0, \Omega^{\varepsilon}},|\widetilde{r}|_{1, \Omega}$,

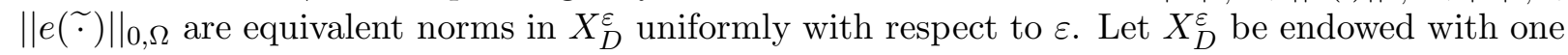
of these norms.

In order to obtain uniform a priori estimates for the sequence $\left\{d^{\varepsilon}\right\}_{\varepsilon>0}$, we use in (15) $d^{\varepsilon}$ as test function, and since $\lambda$ and $A$ are positive, we obtain

$$
2 \mu\left\|e\left(d^{\varepsilon}\right)\right\|_{0, \Omega^{\varepsilon}}^{2} \leq a^{\varepsilon}\left(d^{\varepsilon}, d^{\varepsilon}\right) \leq\left\|f_{S}\right\|_{0, \Omega^{\varepsilon}}\left\|d^{\varepsilon}\right\|_{0, \Omega^{\varepsilon}} .
$$

Now, using extension operator and inequalities (20) and (21) we have

$$
2 \mu\left(\frac{\alpha_{0}}{K}\right)^{2}\left|\widetilde{d^{\varepsilon}}\right|_{1, \Omega}^{2} \leq 2 \mu|| e\left(d^{\varepsilon}\right)\left\|_{0, \Omega^{\varepsilon}}^{2} \leq\right\| f_{S}\left\|\left._{0, \Omega}|| \widetilde{d}^{\varepsilon}\right|_{0, \Omega} \leq \gamma_{0}|| f_{S}\right\|_{0, \Omega}\left|\widetilde{d}^{\varepsilon}\right|_{1, \Omega}
$$

and we have obtained the following

Proposition 2.1 If $f_{S} \in L^{2}(\Omega)^{N}$ then the sequence of solutions $\left\{d^{\varepsilon}\right\}_{\varepsilon>0}$ of (15) is such that

$$
\left|\widetilde{d^{\varepsilon}}\right|_{1, \Omega} \leq C, \quad \forall \varepsilon>0
$$

where $C=C\left(\mu, f_{S}, \Omega, \alpha_{0}, \gamma_{0}, K\right)>0$ is a constant that does not depend on $\varepsilon$ and $\sim$ denotes the extension operator introduced in (19).

\subsection{Two-scale asymptotic expansion}

In this section we use the two-scale asymptotic expansion method to find the homogenized equation in the linear compressible model (13).

In this case we propose the following ansatz for $d^{\varepsilon}$

$$
d^{\varepsilon}(x)=d^{0}(x, x / \varepsilon)+\varepsilon d^{1}(x, x / \varepsilon)+\varepsilon^{2} d^{2}(x, x / \varepsilon)+\ldots,
$$

where $d^{k}, k=0,1,2, \ldots$ is a function such that $d^{k}:(x, y) \in \Omega \times Y \rightarrow d^{k}(x, y) \in \mathbb{R}^{N}$ assumed to be $Y$-periodic in the fast variable $y$.

Remark 2.1 A differential operator $\partial$ applied to such a function now acts as

$$
\partial=\partial_{x}+\frac{1}{\varepsilon} \partial_{y},
$$

where the subscripts stand for the variable in which the differentiation is taken. 
The main idea is to replace (23) in (13) and, by comparison of the powers of $\varepsilon$, we find local differential problems (in the rapid variable $y$ ) parameterized by the macroscopic variable $x$ for $d^{0}$, $d^{1}$ and $d^{2}$. Using (24) it is possible to expand the terms $\sigma\left(d^{\varepsilon}\right) n$ and $\operatorname{div} \sigma\left(d^{\varepsilon}\right)$. These calculations are standard (see [6], for a general overview, and [10] in the case of Stokes equations) so we present here only the expansion of the non-local term in the boundary condition on $\Gamma^{\varepsilon, k}$ in (13), i.e., the integral term

$$
\frac{A}{\varepsilon^{N}} \int_{\Gamma^{\varepsilon, k}} d^{\varepsilon} \cdot n^{\varepsilon} d \gamma
$$

Using the Green formula we have

$$
\frac{A}{\varepsilon^{N}} \int_{\Gamma^{\varepsilon, k}} d^{\varepsilon} \cdot n^{\varepsilon} d \gamma=-\frac{A}{\varepsilon^{N}} \int_{Y_{F}^{\varepsilon, k}} \operatorname{div} d^{\varepsilon} d x=-\frac{A}{\varepsilon^{N}} \int_{Y^{\varepsilon, k}}\left(\operatorname{div} d^{\varepsilon}\right) \chi_{F}^{\varepsilon, k} d x
$$

Note that the minus sign is due to the fact that the normal $n^{\varepsilon}$ points out into $Y_{F}^{\varepsilon, k}$.

The key point is to note that if we use the ansatz (23) in the previous integrals, both macroscopic and microscopic variables are coupled, therefore we have to pay special attention to this non-local term. From (25) the first idea is to consider a constant by cell function defined by

$$
\forall x \in \Omega, \Psi^{\varepsilon}(x)=-\frac{A}{\varepsilon^{N}} \int_{Y^{\varepsilon, k}}\left(\operatorname{div} d^{\varepsilon}\right) \chi_{F}^{\varepsilon, k} d x, \text { if } x \in Y^{\varepsilon, k} .
$$

We would like to find the two scale asymptotic expansion of $\Psi^{\varepsilon}$. Let us denote by $\Pi^{\varepsilon}$ the $L^{2}$ projection operator over the space of constant-by-cell functions. It is defined by

$$
\Pi^{\varepsilon}(v)=\sum_{k \in \mathbb{Z}_{\varepsilon}^{N}} \bar{v}^{\varepsilon, k} \chi^{\varepsilon, k}, \quad v \in L^{2}(\Omega)
$$

where $\bar{v}^{\varepsilon, k}$ is the mean value of $v$ in cell $Y^{\varepsilon, k}$, that is

$$
\bar{v}^{\varepsilon, k}=\frac{1}{\varepsilon^{N}|Y|} \int_{Y^{\varepsilon, k}} v\left(x^{\prime}\right) d x^{\prime} .
$$

Using (26), (27) and (28), we have

$$
\forall x \in \Omega, \Psi^{\varepsilon}(x)=-|Y| A \Pi^{\varepsilon}\left(\operatorname{div} d^{\varepsilon} \chi_{F}^{\varepsilon}\right)(x), \text { if } x \in Y^{\varepsilon, k},
$$

where $\chi_{F}^{\varepsilon}$ is defined in 5. Hence, we are led to study the two scale asymptotic expansion of $\Pi^{\varepsilon}\left(\operatorname{div} d^{\varepsilon} \chi_{F}^{\varepsilon}\right)$. To do this we will first analyze, the expansion of $\Pi^{\varepsilon}(\varphi)$ with $\varphi \in C^{\infty}\left(\bar{\Omega} ; L_{\#}^{\infty}(Y)\right)$, and then, apply this result to $\varphi=\left(\operatorname{div} d^{\varepsilon}\right) \chi_{F}^{\varepsilon}$ with $d^{\varepsilon}$ given by (23). For any $x \in Y^{\varepsilon, k}$

$$
\Pi^{\varepsilon}(\varphi)(x)=\frac{1}{\varepsilon^{N}|Y|} \int_{Y^{\varepsilon, k}} \varphi\left(x^{\prime}, \frac{x^{\prime}}{\varepsilon}\right) d x^{\prime} .
$$

Since $x^{\prime} \in Y^{\varepsilon, k}$ we have $x^{\prime}=x^{\varepsilon, k}+\varepsilon y^{\prime}$ with $y^{\prime} \in Y\left(x^{\varepsilon, k}=\varepsilon(k+0)\right.$ denotes the translation and homothetic transformation of the center of the reference cell $Y$ ). Using this change of variables and the periodicity of $\varphi$ in the second argument, we have

$$
\Pi^{\varepsilon}(\varphi)(x)=\int_{Y} \varphi\left(x^{\varepsilon, k}+\varepsilon y^{\prime}, y^{\prime}\right) d y^{\prime}
$$


Performing a formal Taylor expansion of $\varphi\left(\cdot, y^{\prime}\right)$ around the point $x$ we obtain (note that $x=$ $x^{\varepsilon, k}+\varepsilon y$ with $y \in Y$, so $\left.x^{\prime}-x=\varepsilon\left(y^{\prime}-y\right)\right)$ :

$$
\begin{aligned}
\frac{1}{|Y|} \int_{Y} \varphi\left(x^{\varepsilon, k}+\varepsilon y^{\prime}, y^{\prime}\right) d y^{\prime}= & \frac{1}{|Y|} \int_{Y}\left(\varphi\left(x, y^{\prime}\right)+\varepsilon\left(y^{\prime}-y\right) \cdot \nabla_{x} \varphi\left(x, y^{\prime}\right)\right. \\
& \left.+\varepsilon^{2}\left(y^{\prime}-y\right)^{T} D_{x}^{2} \varphi\left(x, y^{\prime}\right)\left(y^{\prime}-y\right)+O\left(\varepsilon^{3}\right)\right) d y^{\prime} .
\end{aligned}
$$

Hence, $\Pi^{\varepsilon}(\varphi)$ can be written as

$$
\Pi^{\varepsilon}(\varphi)(x)=\bar{\varphi}^{0}(x)+\varepsilon \bar{\varphi}^{-1}(x, y)+\varepsilon^{2} \bar{\varphi}^{2}(x, y)+\ldots,
$$

where

$$
\begin{gathered}
\bar{\varphi}^{0}(x)=\frac{1}{|Y|} \int_{Y} \varphi\left(x, y^{\prime}\right) d y^{\prime} \\
\bar{\varphi}^{-1}(x, y)=\frac{1}{|Y|} \int_{Y}\left(y^{\prime}-y\right) \cdot \nabla_{x} \varphi\left(x, y^{\prime}\right) d y^{\prime}
\end{gathered}
$$

and

$$
\bar{\varphi}^{2}(x, y)=\frac{1}{|Y|} \int_{Y}\left(y^{\prime}-y\right)^{T} D_{x}^{2} \varphi\left(x, y^{\prime}\right)\left(y^{\prime}-y\right) d y^{\prime} .
$$

Note that the first term $\bar{\varphi}^{0}$ is nothing but the $L^{2}$-weak limit of $\Pi^{\varepsilon}(\varphi)$ as $\varepsilon$ goes to 0 .

Finally, since

$$
\begin{aligned}
\operatorname{div}^{\varepsilon}(x)= & \varepsilon^{-1} \operatorname{div}_{y} d^{0}(x, x / \varepsilon)+\operatorname{div}_{x} d^{0}(x, x / \varepsilon)+\operatorname{div}_{y} d^{1}(x, x / \varepsilon) \\
& +\varepsilon\left(\operatorname{div}_{x} d^{1}(x, x / \varepsilon)+\operatorname{div}_{y} d^{2}(x, x / \varepsilon)\right)+\ldots,
\end{aligned}
$$

we obtain that

$$
\begin{aligned}
\Psi^{\varepsilon}(x)= & -A\left(\varepsilon^{-1} \int_{Y_{F}} \operatorname{div}_{y} d^{0}\left(x, y^{\prime}\right) d y^{\prime}\right. \\
& +\int_{Y_{F}}\left(\operatorname{div}_{x} d^{0}\left(x, y^{\prime}\right)+\operatorname{div}_{y} d^{1}\left(x, y^{\prime}\right)\right) d y^{\prime} \\
& +\int_{Y_{F}}\left(y^{\prime}-y\right) \nabla_{x} \operatorname{div}_{y} d^{0}\left(x, y^{\prime}\right) d y^{\prime} \\
& +\varepsilon\left[\int_{Y_{F}}\left(\operatorname{div}_{x} d^{1}\left(x, y^{\prime}\right)+\operatorname{div}_{y} d^{2}\left(x, y^{\prime}\right)\right) d y^{\prime}\right. \\
& +\int_{Y_{F}}\left(y^{\prime}-y\right) \nabla_{x}\left(\operatorname{div}_{x} d^{0}\left(x, y^{\prime}\right)+\operatorname{div}_{y} d^{1}\left(x, y^{\prime}\right)\right) d y^{\prime} \\
& \left.\left.+\int_{Y_{F}}\left(y^{\prime}-y\right)^{T} D_{x}^{2} \operatorname{div}_{y} d^{0}\left(x, y^{\prime}\right)\left(y^{\prime}-y\right) d y^{\prime}\right]+\ldots\right) .
\end{aligned}
$$

In (29) we can use Green formula in some integrals of this expansion, in the following way

$$
\int_{Y_{F}} \operatorname{div}_{y} d^{k}\left(x, y^{\prime}\right) d y^{\prime}=-\int_{\Gamma} d^{k} \cdot n
$$

The second integral is an integral with respect to the fast variable. In all what follows any surface integral over $\Gamma$ has to be understood as an integral with respect to the fast variable. 
Replacing these expansions in (13) and by comparison of the powers of $\varepsilon$ we find the first local problem in $Y_{S}$ :

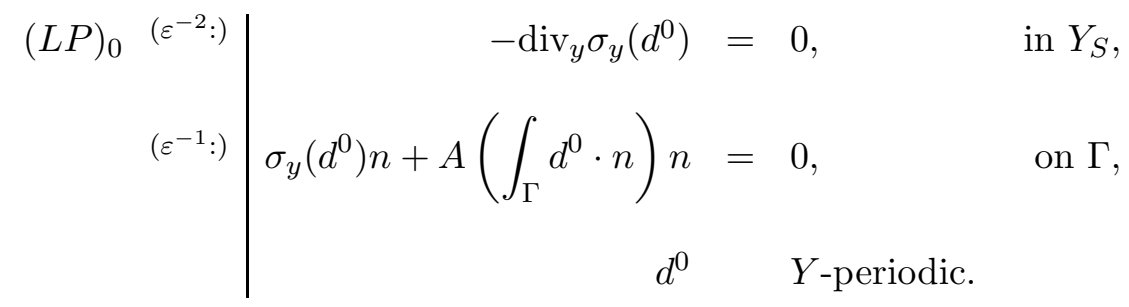

It is not difficult to see that the unique solution to this problem is, up to an additive constant, the null function. Hence $d^{0}=d^{0}(x)$. Taking into account that $d^{0}$ does not depend on $y$, the next local problem reads

$$
\begin{array}{r|rl}
(L P)_{1} \quad\left(\varepsilon^{-1}:\right) & & \text { in } Y_{S} \\
-\operatorname{div}_{y} \sigma_{y}\left(d^{1}\right) & = & \\
\sigma_{y}\left(d^{1}\right) n+A\left(\int_{\Gamma} d^{1} \cdot n\right) n= & -\sigma_{x}\left(d^{0}\right) n \\
& +A\left(\left|Y_{F}\right| \operatorname{div}_{x} d^{0}\right) n, & \text { on } \Gamma \\
d^{1} & & Y \text {-periodic. }
\end{array}
$$

This problem gives $d^{1}$ knowing $d^{0}$. In order to obtain the equation satisfied by $d^{0}$ we write the differential problem for $d^{2}$ :

$$
(L P)_{2}\left(\varepsilon^{0}:\right) \mid \begin{aligned}
-\operatorname{div}_{y} \sigma_{y}\left(d^{2}\right)=\operatorname{div}_{x} \sigma_{y}\left(d^{1}\right)+ & \operatorname{div}_{x} \sigma_{x}\left(d^{0}\right) \\
& +\operatorname{div}_{y} \sigma_{x}\left(d^{1}\right)+f_{S}, \\
\sigma_{y}\left(d^{2}\right) n+A\left(\varepsilon_{\Gamma} d^{2} \cdot n\right) n= & -\sigma_{x}\left(d^{1}\right) n+A\left(\int_{Y_{F}} \operatorname{div}_{x} d^{1} d y^{\prime}\right) n \\
+ & A\left(\int_{Y_{F}} y^{\prime} \cdot \nabla_{x}\left(\operatorname{div}_{x} d^{0}+\operatorname{div}_{y} d^{1}\right) d y^{\prime}\right) n \\
& -A\left(\int_{Y_{F}} y \cdot \nabla_{x}\left(\operatorname{div}_{x} d^{0}+\operatorname{div}_{y} d^{1}\right) d y^{\prime}\right) n, \quad \text { on } \Gamma, \\
& d^{2} Y \text {-periodic, }
\end{aligned}
$$

where we have used (29), (30) and again the fact that $d^{0}=d^{0}(x)$.

The local problems $(L P)_{n}(n=0,1,2)$ are particular instances of the following problem: find $\chi$ defined over $Y_{S}$ such that

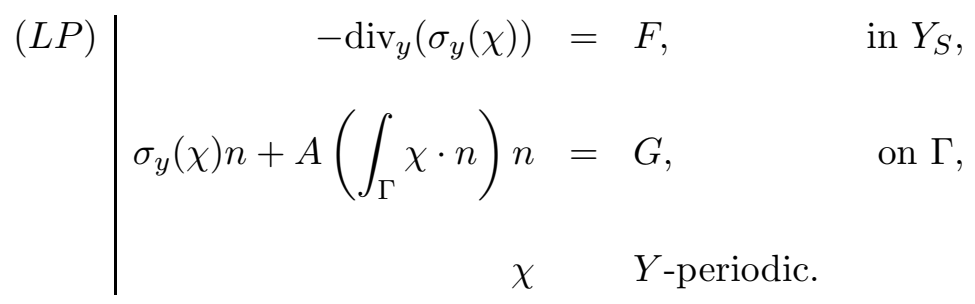


In order to have existence and uniqueness of a solution for the problem $(L P)$ and since $\int_{\Gamma} n=0$, the compatibility condition that must be satisfied (see [10]) reads:

$$
\int_{Y_{S}} F d y+\int_{\Gamma} G=0
$$

It is easy to see that $(31)$ is satisfied in $(L P)_{0}$ and $(L P)_{1}$. The condition $(31)$ applied to $(L P)_{2}$ provides a further relation between $d^{0}$ and $d^{1}$. Indeed, we obtain

$$
\begin{aligned}
-\left|Y_{S}\right| \operatorname{div}_{x}\left(\sigma_{x}\left(d^{0}\right)+\frac{1}{\left|Y_{S}\right|} \int_{Y_{S}} \sigma_{y}\left(d^{1}\right)\right)= \\
=\left|Y_{S}\right| f_{S}-A \int_{\Gamma}\left(y \cdot \int_{Y_{F}} \nabla_{x}\left(\operatorname{div}_{x} d^{0}+\operatorname{div}_{y} d^{1}\right) d y^{\prime}\right) n
\end{aligned}
$$

where we have used the fact that $\int_{\Gamma} H(x) n=H(x) \int_{\Gamma} n=0$ and $\int_{Y_{S}} \operatorname{div}_{y} \sigma_{x}\left(d^{1}\right) d y=\int_{\Gamma} \sigma_{x}\left(d^{1}\right) n$ that results from the $Y$-periodicity of $d^{1}$ and the Green formula. Recalling that $d^{0}$ does not depend on $y$ and that $d^{1}$ is periodic with respect to $y$ the left hand side of (32) reads

$$
\begin{aligned}
\int_{\Gamma}\left(y \cdot \int_{Y_{F}} \nabla_{x}\right. & \left.\left(\operatorname{div}_{x} d^{0}+\operatorname{div}_{y} d^{1}\right) d y^{\prime}\right) n= \\
& =\int_{\Gamma} y \cdot\left(\left|Y_{F}\right| \nabla_{x} \operatorname{div}_{x} d^{0}-\nabla_{x} \int_{Y_{S}} \operatorname{div}_{y} d^{1} d y^{\prime}\right) n \\
& =\left(\int_{\Gamma} y \otimes n\right)\left(\left|Y_{F}\right| \nabla_{x} \operatorname{div}_{x} d^{0}-\nabla_{x} \int_{Y_{S}} \operatorname{div}_{y} d^{1} d y^{\prime}\right)
\end{aligned}
$$

where the tensorial notation $b \otimes n$ stands for the $N \times N$ matrix defined by $(b \otimes n)_{i j}=n_{i} b_{j}$, so that, for any $a, b \in \mathbb{R}^{N}$,

$$
(a \cdot b) n=(b \otimes n) a .
$$

Finally, we note that

$$
\int_{\Gamma} n_{i} y_{j}=-\int_{Y_{F}} \operatorname{div}_{y}\left(y_{j} e^{i}\right) d y=-\left|Y_{F}\right| \delta_{j i}
$$

where $e^{i}$ is the $i^{\text {th }}$ canonical unit vector in $\mathbb{R}^{N}$, therefore

$$
\begin{aligned}
\int_{\Gamma}\left(y \cdot \int_{Y_{F}} \nabla_{x}\left(\operatorname{div}_{x} d^{0}+\operatorname{div}_{y} d^{1}\right) d y^{\prime}\right) n= \\
=-\left|Y_{F}\right|\left(\left|Y_{F}\right| \nabla_{x} \operatorname{div}_{x} d^{0}-\nabla_{x} \int_{Y_{S}} \operatorname{div}_{y} d^{1} d \bar{y}\right) .
\end{aligned}
$$

Replacing in (32), reordering, and using the notations $\sigma^{0}$ and $\sigma^{1}$ defined by

$$
\sigma^{0}(\cdot)=2 \mu e(\cdot)+\lambda_{0} \operatorname{div}(\cdot) I, \text { with } \lambda_{0}=\lambda+A \frac{\left|Y_{F}\right|^{2}}{\left|Y_{S}\right|},
$$

and

we obtain

$$
\sigma^{1}(\cdot)=2 \mu e(\cdot)+\lambda_{1} \operatorname{div}(\cdot) I, \text { with } \lambda_{1}=\lambda-A\left|Y_{F}\right|,
$$

$$
-\operatorname{div}_{x}\left(\sigma_{x}^{0}\left(d^{0}\right)+\frac{1}{\left|Y_{S}\right|} \int_{Y_{S}} \sigma_{y}^{1}\left(d^{1}\right) d y\right)=f_{S} .
$$

Therefore (34), (35) and (36) is the formal homogenized differential problem, as $\epsilon$ goes to 0 , of the elastic incompressible media with gaseous bubbles (13). 


\subsection{Two-scale convergence method}

In this section we apply the two-scale homogenization method in order to mathematically justify the homogenized limit problem (34), (35) and (36). The main idea of this method was introduced firstly by G. Nguetseng in 1989 [28] and then generalized and applied to several important cases by G. Allaire in 1992 [3], including the case of perforated domains. The two-scale method applied to the Stokes equations with non-slip boundary conditions (this case coincides in the stationary case to incompressible elasticity) has been studied in [4].

We recall the following definition (see [3]):

Definition 2.1 We say that $\left\{v^{\varepsilon}\right\} \subset L^{2}(\Omega)$ two-scale converges to some function $v \in L^{2}\left(\Omega ; L_{\#}^{2}(Y)\right)$ (and we will denote it by $v^{\varepsilon} \stackrel{2}{\rightarrow} v$ ) if

$$
\lim _{\varepsilon \rightarrow 0} \int_{\Omega} v^{\varepsilon}(x) \psi\left(x, \frac{x}{\varepsilon}\right) d x=\int_{\Omega} \int_{Y} v(x, y) \psi(x, y) d y d x,
$$

for all admissible function $\psi \in L^{2}\left(\Omega ; C_{\#}^{\infty}(Y)\right)$ or $\psi \in C^{\infty}\left(\bar{\Omega} ; L_{\#}^{2}(Y)\right)$.

The same definition can be given for $\left\{v^{\varepsilon}\right\} \subset L^{2}(\Omega)^{N}$ with a two-scale limit $v$ in $L^{2}\left(\Omega ; L_{\#}^{2}(Y)\right)^{N}$ or for $\left\{V^{\varepsilon}\right\} \subset L^{2}(\Omega)^{N \times N}$ with a two-scale limit $V$ in $L^{2}\left(\Omega ; L_{\#}^{2}(Y)\right)^{N \times N}$ by changing the product by a dot product for vectors or the tensorial product for matrices, respectively.

From the a priori estimates of Proposition 2.1, it is well known that the following two-scale convergence result holds (see [3]):

Proposition 2.2 Let $\left\{\widetilde{d}^{\varepsilon}\right\}_{\varepsilon>0}$ be the sequence of extended weak solution of (13), then there exist $d^{0} \in X_{D}$ and $d^{1} \in L^{2}\left(\Omega ; H_{\#}^{1}(Y) / \mathbb{R}\right)^{N}$ such that, as $\varepsilon$ tends to 0 ,

$$
\begin{aligned}
& \widetilde{d}^{\varepsilon} \stackrel{2}{\rightarrow} d^{0}, \\
& \nabla \widetilde{d^{\varepsilon}} \stackrel{2}{\rightarrow} \nabla_{x} d^{0}+\nabla_{y} d^{1}, \\
& \operatorname{div} \widetilde{d}^{\varepsilon} \stackrel{2}{\longrightarrow} \operatorname{div}_{x} d^{0}+\operatorname{div}_{y} d^{1}, \\
& e\left(\widetilde{d^{\varepsilon}}\right) \stackrel{2}{\longrightarrow} e_{x}\left(d^{0}\right)+e_{y}\left(d^{1}\right),
\end{aligned}
$$

where the extension operator $\sim$ was introduced in (19).

The main difficulty when taking the limit of $a\left(d^{\varepsilon}, \varphi^{\varepsilon}\right)$ is to deal with the product of boundary integrals, that is,

$$
a_{\Gamma}^{\varepsilon}\left(d^{\varepsilon}, \varphi^{\varepsilon}\right)=\frac{A}{\varepsilon^{N}} \sum_{k \in \mathbb{Z}_{\varepsilon}^{N}}\left(\int_{\Gamma^{\varepsilon, k}} d^{\varepsilon} \cdot n d \gamma\right)\left(\int_{\Gamma^{\varepsilon, k}} \varphi^{\varepsilon} \cdot n d \gamma\right) .
$$

Following [5], a way to overcome this difficulty is to rewrite this term using the already defined $L^{2}$-projection operator over the constant by cell functions (see $(27)$ ).

Indeed by noticing that $Y^{\varepsilon, k} \cap Y^{\varepsilon, l}=\emptyset$, for $k \neq l$, it is easy to see that for all $u, v \in L^{2}(\Omega)$

$$
\int_{\Omega} \Pi^{\varepsilon}(u) \Pi^{\varepsilon}(v) d x=\frac{1}{\varepsilon^{N}|Y|} \sum_{k \in \mathbb{Z}_{\varepsilon}^{N}}\left(\int_{Y^{\varepsilon, k}} u d x\right)\left(\int_{Y^{\varepsilon, k}} v d x\right) .
$$

Then, since $\Pi^{\varepsilon}$ is a $L^{2}$-projection operator, we also have that

$$
\int_{\Omega} \Pi^{\varepsilon}(u) \Pi^{\varepsilon}(v) d x=\int_{\Omega} u \Pi^{\varepsilon}(v) d x=\int_{\Omega} \Pi^{\varepsilon}(u) v d x, \quad \forall u, v \in L^{2}(\Omega) .
$$


Finally, using the extension operator we have

$$
\int_{\Gamma^{\varepsilon, k}} d^{\varepsilon} \cdot n d \gamma=-\int_{Y_{F}^{\varepsilon, k}} \operatorname{div} \widetilde{d^{\varepsilon}} d x=-\int_{Y^{\varepsilon, k}} \chi_{F}^{\varepsilon, k} \operatorname{div} \widetilde{d}^{\varepsilon} d x
$$

Therefore, thanks to (5), (42), (43) and (44) we have that

$$
a_{\Gamma}^{\varepsilon}\left(d^{\varepsilon}, \varphi^{\varepsilon}\right)=A \int_{\Omega}\left(\chi_{F}^{\varepsilon} \operatorname{div} \widetilde{d^{\varepsilon}}\right) \Pi^{\varepsilon}\left(\chi_{F}^{\varepsilon} \operatorname{div} \widetilde{\varphi^{\varepsilon}}\right) d x .
$$

The following strong convergence result, that we will use when passing to the two-scale limit, holds

Lemma 2.3 Let $\Pi^{\varepsilon}$ be the projection operator defined in $(27)$. Then for all $\varphi \in C^{\infty}\left(\bar{\Omega} ; C_{\#}^{\infty}(Y)\right)$

$$
\Pi^{\varepsilon}\left(\chi_{F}^{\varepsilon} \varphi\left(\cdot, \frac{\dot{\varepsilon}}{\varepsilon}\right)\right)(x) \longrightarrow \frac{1}{|Y|} \int_{Y} \varphi(x, y) \chi_{F}(y) d y \quad L^{2}(\Omega) \text {-strongly. }
$$

Proof. Let us take a fixed $x \in \Omega$. There exists $(\varepsilon, k)$ (actually may be for $\varepsilon$ small enough if $x$ is close to the boundary) such that $x$ belongs to $Y^{\varepsilon, k}$. Thus

$$
\Pi^{\varepsilon}\left(\chi_{F}^{\varepsilon} \varphi\left(\cdot, \frac{\dot{⿰}}{\varepsilon}\right)\right)(x)=\frac{1}{\varepsilon^{N}|Y|} \int_{Y_{F}^{\varepsilon, k}} \varphi\left(x^{\prime}, \frac{x^{\prime}}{\varepsilon}\right) d x^{\prime} .
$$

The same calculation we did before when writing the two scale asymptotic expansion gives

$$
\Pi^{\varepsilon}\left(\chi_{F}^{\varepsilon} \varphi\left(\cdot, \frac{\dot{\varepsilon}}{\varepsilon}\right)\right)(x)=\frac{1}{|Y|} \int_{Y_{F}}\left(\varphi\left(x, y^{\prime}\right)+\varepsilon\left(y^{\prime}-y_{\varepsilon}\right) \cdot \nabla_{x} \varphi\left(x, y^{\prime}\right)+O\left(\varepsilon^{2}\right)\right) d y^{\prime},
$$

with $y_{\varepsilon}=\frac{x-x^{\varepsilon, k}}{\varepsilon} \in Y$.

From the previous expansion and since $y_{\varepsilon}$ is bounded we obtain, as $\varepsilon$ tends to zero, that

$$
\Pi^{\varepsilon}\left(\chi_{F}^{\varepsilon} \varphi\left(\cdot, \frac{\dot{\varepsilon}}{\varepsilon}\right)\right)(x) \longrightarrow \frac{1}{|Y|} \int_{Y_{F}} \varphi(x, y) d y, \quad \text { a.e. in } \Omega .
$$

Therefore the result follows from dominated convergence since it is clear that

$$
\left|\Pi^{\varepsilon}\left(\chi_{F}^{\varepsilon} \varphi\left(\cdot, \frac{\dot{x}}{\varepsilon}\right)\right)(x)\right| \leq \frac{\left|Y_{F}\right|}{|Y|} \sup _{x \in \Omega, y \in Y}|\varphi(x, y)|, \quad \forall x \in \Omega .
$$

Now, we are able to prove the following convergence result:

Theorem 2.4 Let $\left\{d^{\varepsilon}\right\}_{\varepsilon>0}$ be the sequence of weak solutions of $(15)$. Then the sequences $\left\{\widetilde{d}^{\varepsilon}\right\}_{\varepsilon>0}$, $\left\{\nabla \widetilde{d^{\varepsilon}}\right\}_{\varepsilon>0}$ two-scale converge to $d^{0}$ and $\nabla_{x} d^{0}+\nabla_{y} d^{1}$ where $\left(d^{0}, d^{1} \chi_{S}\right) \in X_{D} \times L^{2}\left(\Omega ; H_{\#}^{1}\left(Y_{S}\right) / \mathbb{R}\right)^{N}$ is the unique solution of the following variational two-scale homogenized problem

$$
\begin{aligned}
& 2 \mu \int_{\Omega} \int_{Y_{S}}\left(e_{x}\left(d^{0}\right)+e_{y}\left(d^{1}\right)\right):\left(e_{x}(\phi)+e_{y}\left(\phi^{1}\right)\right) d y d x \\
& +\lambda \int_{\Omega} \int_{Y_{S}}\left(\operatorname{div}_{x} d^{0}+\operatorname{div}_{y} d^{1}\right)\left(\operatorname{div}_{x} \phi+\operatorname{div}_{y} \phi^{1}\right) d y d x \\
& +A \int_{\Omega}\left(\left|Y_{F}\right| \operatorname{div}_{x} d^{0}-\int_{\Gamma} d^{1} \cdot n\right)\left(\left|Y_{F}\right| \operatorname{div}_{x} \phi-\int_{\Gamma} \phi^{1} \cdot n\right) d x \\
& =\left|Y_{S}\right| \int_{\Omega} f_{S} \cdot \phi d x,
\end{aligned}
$$

for all $\left(\phi, \phi^{1}\right) \in X_{D} \times L^{2}\left(\Omega ; H_{\#}^{1}\left(Y_{S}\right) / \mathbb{R}\right)^{N}$. 
Proof. In order to take the two-scale limit in the variational formulation (15) we choose test functions of the form

$$
\varphi^{\varepsilon}(x, y)=\left(\phi(x)+\varepsilon \phi^{1}(x, y)\right),
$$

where $\phi \in C^{\infty}(\bar{\Omega})^{N}$, such that $\phi=0$ on $\Gamma_{D}$, and $\phi^{1} \in C^{\infty}\left(\bar{\Omega} ; C_{\#}^{\infty}(Y)\right)^{N}$. These test functions are admissible for the two-scale convergence definition since $\varphi^{\varepsilon} \in C^{\infty}\left(\bar{\Omega} ; L_{\#}^{2}(Y)\right)^{N}$ (see [3]).

Using the extension operator $\sim$ we can write all the integrals over $\Omega^{\varepsilon}$ in the variational formulation (15) as integrals over $\Omega$. That is

$$
2 \mu \int_{\Omega} e\left(\widetilde{d^{\varepsilon}}\right): e\left(\varphi^{\varepsilon}\right) \chi_{S}^{\varepsilon} d x+\lambda \int_{\Omega} \operatorname{div} \widetilde{d}^{\varepsilon} \operatorname{div} \varphi^{\varepsilon} \chi_{S}^{\varepsilon} d x+a_{\Gamma}^{\varepsilon}\left(\widetilde{d}^{\varepsilon}, \varphi^{\varepsilon}\right)=\int_{\Omega} f_{S} \cdot \varphi^{\varepsilon} \chi_{S}^{\varepsilon} d x .
$$

It is easy to see, using (41) in Proposition 2.2, that we can pass to the limit as $\varepsilon$ goes to 0 in the first and second term in the left-hand side of (48). In fact, we have

$$
2 \mu \int_{\Omega} e\left(\widetilde{d}^{\varepsilon}\right): e\left(\varphi^{\varepsilon}\right) \chi_{S}^{\varepsilon} d x \longrightarrow 2 \mu \int_{\Omega} \int_{Y_{S}}\left(e_{x}\left(d^{0}\right)+e_{y}\left(d^{1}\right)\right):\left(e_{x}(\phi)+e_{y}\left(\phi^{1}\right)\right) d y d x,
$$

and

$$
\lambda \int_{\Omega} \operatorname{div} \widetilde{d^{\varepsilon}} \operatorname{div} \varphi^{\varepsilon} \chi_{S}^{\varepsilon} d x \longrightarrow \lambda \int_{\Omega} \int_{Y_{S}}\left(\operatorname{div}_{x} d^{0}+\operatorname{div}_{y} d^{1}\right)\left(\operatorname{div}_{x} \phi+\operatorname{div}_{y} \phi^{1}\right) d y d x
$$

In order to pass to the limit in the third term as already announced we use (45) and again Proposition 2.2. However, we have to verify that $\Pi^{\varepsilon}\left(\chi_{F}^{\varepsilon} \operatorname{div} \varphi^{\varepsilon}\right)$ converges $L^{2}$-strongly and we have to identify its limit. This is done by using Lemma 2.3 with $\operatorname{div} \varphi^{\varepsilon}$ as test function. Since $\operatorname{div} \varphi^{\varepsilon}=\operatorname{div}\left(\phi(x)+\varepsilon \phi^{1}(x, x / \varepsilon)\right)=\operatorname{div}_{x} \phi+\operatorname{div}_{y} \phi^{1}+\varepsilon \operatorname{div}_{x} \phi^{1}$, we obtain that

$$
\Pi^{\varepsilon}\left(\chi_{F}^{\varepsilon} \operatorname{div} \varphi^{\varepsilon}\right) \longrightarrow \frac{1}{|Y|} \int_{Y}\left(\operatorname{div}_{x} \phi+\operatorname{div}_{y} \phi^{1}\right) \chi_{F} d y, \quad \text { strongly in } L^{2}(\Omega) .
$$

Now, thanks to (40) in Proposition 2.2 and the definition of two-scale convergence we have

$$
\operatorname{div} \widetilde{d}^{\varepsilon} \chi_{F}^{\varepsilon} \rightarrow \int_{Y}\left(\operatorname{div}_{x} d^{0}+\operatorname{div}_{y} d^{1}\right) \chi_{F} d y, \text { weakly in } L^{2}(\Omega) .
$$

Hence, combining the above $L^{2}$-strong and $L^{2}$-weak convergences, we can pass to the limit in (45) to obtain that

$$
a_{\Gamma}^{\varepsilon}\left(\widetilde{d^{\varepsilon}}, \varphi^{\varepsilon}\right) \longrightarrow A \int_{\Omega}\left(\int_{Y_{F}}\left(\operatorname{div}_{x} d^{0}+\operatorname{div}_{y} d^{1}\right) d y\right)\left(\int_{Y_{F}}\left(\operatorname{div}_{x} \phi+\operatorname{div}_{y} \phi^{1}\right) d y\right) d x .
$$

Note that this limit can be rewritten as

$$
A \int_{\Omega}\left(\left|Y_{F}\right| \operatorname{div}_{x} d^{0}-\int_{\Gamma} d^{1} \cdot n\right)\left(\left|Y_{F}\right| \operatorname{div}_{x} \phi-\int_{\Gamma} \phi^{1} \cdot n\right) d x
$$

Finally, we pass to the limit in the right-hand side of (48),

$$
\ell\left(\varphi^{\varepsilon}\right)=\int_{\Omega}\left(f_{S} \chi_{S}^{\varepsilon}\right) \cdot \varphi^{\varepsilon} \rightarrow\left|Y_{S}\right| \int_{\Omega} f_{S} \cdot \phi d x
$$

Consequently, we can pass to the limit in (15) and obtain (46), for all $\left(\phi, \phi^{1}\right) \in\left\{\psi \in C^{\infty}(\bar{\Omega})^{N}\right.$ : $\left.\left.\psi\right|_{\Gamma_{D}}=0\right\} \times C^{\infty}\left(\bar{\Omega} ; C_{\#}^{\infty}(Y)\right)^{N}$ which is dense in $X_{D} \times L^{2}\left(\Omega ; H_{\#}^{1}\left(Y_{S}\right) / \mathbb{R}\right)^{N}$.

What remains to be proven is the uniqueness of $\left(d^{0}, d^{1} \chi_{S}\right) \in X_{D} \times L^{2}\left(\Omega ; H_{\#}^{1}\left(Y_{S}\right) / \mathbb{R}\right)^{N}$ solution of the two-scale weak limit problem (46). It is not difficult to check that the left hand side of (46) is $X_{D} \times L^{2}\left(\Omega ; H_{\#}^{1}\left(Y_{S}\right) / \mathbb{R}\right)^{N}$-coercive as a consequence of the following lemma: 
Lemma 2.5 For any $\left(d^{0}, d^{1}\right) \in X_{D} \times L^{2}\left(\Omega ; H_{\#}^{1}\left(Y_{S}\right) / \mathbb{R}\right)^{N}$ there exists a constant $C>0$ such that

$$
\begin{gathered}
\left\|d^{0}\right\|_{L^{2}(\Omega)^{N}}+\left\|d^{1}\right\|_{L^{2}\left(\Omega ; L_{\#}^{2}\left(Y_{S}\right) / \mathbb{R}\right)^{N}}+\left\|\nabla_{x} d^{0}\right\|_{L^{2}(\Omega)^{N \times N}}+\left\|\nabla_{y} d^{1}\right\|_{L^{2}\left(\Omega \times Y_{S}\right)^{N \times N}} \\
\leq C\left\|e_{x}\left(d^{0}\right)+e_{y}\left(d^{1}\right)\right\|_{L^{2}\left(\Omega \times Y_{S}\right)^{N \times N}}
\end{gathered}
$$

This lemma states that $\left\|e_{x}\left(d^{0}\right)+e_{y}\left(d^{1}\right)\right\|_{L^{2}\left(\Omega \times Y_{S}\right)^{N \times N}}$ is a norm for the Hilbert space $X_{D} \times$ $L^{2}\left(\Omega ; H_{\#}^{1}\left(Y_{S}\right) / \mathbb{R}\right)^{N}$. The proof is given in Appendix A.

It is now possible to identify two differential problems from the variational two-scale problem (46). One in the macroscopic variable, and the other one in the microscopic scale. This is done by using special test functions.

First, by taking $\phi=0$ and integrating by parts in $Y_{F}$ in (46) we obtain that $\forall \phi^{1} \in C^{\infty}\left(\bar{\Omega} ; C_{\#}^{\infty}(Y)\right)^{N}$,

$$
\begin{aligned}
& 2 \mu \int_{\Omega} \int_{Y_{S}}\left(e_{x}\left(d^{0}\right)+e_{y}\left(d^{1}\right)\right): e_{y}\left(\phi^{1}\right) d y d x \\
& +\lambda \int_{\Omega} \int_{Y_{S}}\left(\operatorname{div}_{x} d^{0}+\operatorname{div}_{y} d^{1}\right) \operatorname{div}_{y} \phi^{1} d y d x \\
& -A\left|Y_{F}\right| \int_{\Omega} \operatorname{div}_{x} d^{0}\left(\int_{\partial Y_{F}} \phi^{1} \cdot n\right) d x \\
& +A \int_{\Omega}\left(\int_{\partial Y_{F}} d^{1} \cdot n\right)\left(\int_{\partial Y_{F}} \phi^{1} \cdot n\right) d x=0 .
\end{aligned}
$$

Using $\phi^{1} \in \mathcal{D}\left(\Omega ; C_{\#}^{\infty}(Y)\right)^{N}$ such that $\phi^{1}=0$ for $y \in Y_{F}$ in (50), and after an integration by parts, we obtain that

$$
-\operatorname{div}_{y} \sigma_{y}\left(d^{1}\right)=0, \quad \text { in } \Omega \times Y_{S} .
$$

Then, multiplying (51) by $\phi^{1} \in \mathcal{D}\left(\Omega ; C_{\#}^{\infty}(Y)\right)^{N}$ integrating by parts and comparing again with (50) with the same test functions, we obtain the following boundary condition

$$
\left(\sigma_{y}\left(d^{1}\right)+A\left(\int_{\partial Y_{F}} d^{1} \cdot n\right)\right) n=A\left|Y_{F}\right|\left(\operatorname{div}_{x} d^{0}\right) n-\sigma_{x}\left(d^{0}\right) n, \text { on } \Omega \times \partial Y_{F}
$$

By taking now $\phi^{1}=0$ in (46) and also integrating by parts twice in $Y_{F}$ we obtain for all $\phi \in C^{\infty}(\bar{\Omega})$ that

$$
\begin{aligned}
& 2 \mu \int_{\Omega} \int_{Y_{S}}\left(e_{x}\left(d^{0}\right)+e_{y}\left(d^{1}\right)\right): e_{x}(\phi) d y d x+ \\
& \lambda \int_{\Omega} \int_{Y_{S}}\left(\operatorname{div}_{x} d^{0}+\operatorname{div}_{y} d^{1}\right) \operatorname{div}_{x} \phi d y d x+ \\
& +A\left|Y_{F}\right|^{2} \int_{\Omega} \operatorname{div}_{x} d^{0} \operatorname{div}_{x} \phi d x-A\left|Y_{F}\right| \int_{\Omega}\left(\int_{Y_{S}} \operatorname{div}_{y} d^{1} d x\right) \operatorname{div}_{x} \phi d x \\
& =\left|Y_{S}\right| \int_{\Omega} f_{S} \cdot \phi d x .
\end{aligned}
$$

By using the notations of the stress tensors $\sigma^{0}$ and $\sigma^{1}$ introduced in (34), (35) and taking $\phi \in \mathcal{D}(\Omega)^{N}$ in (53) we recover (36):

$$
-\operatorname{div} \sigma_{x}^{0}\left(d^{0}\right)-\operatorname{div}\left(\frac{1}{\left|Y_{S}\right|} \int_{Y_{S}} \sigma_{y}^{1}\left(d^{1}\right) d y\right)=f_{S}, \quad \text { in } \Omega .
$$


Then, by multiplying (54) by $\phi \in H^{1}(\Omega), \phi=0$ if $x \in \Gamma_{D}$ (that is $\phi \in X_{D}$ ) and integrating by parts, after comparing with (53) with the same test functions we obtain the following boundary condition for $(54)$

$$
\sigma_{x}^{0}\left(d^{0}\right) n+\left(\frac{1}{\left|Y_{S}\right|} \int_{Y_{S}} \sigma_{y}^{1}\left(d^{1}\right) n d y\right)=0, \quad \text { on } \Gamma_{N} .
$$

Therefore, $\left(d^{0}, d^{1}\right)$ is solution of the following coupled differential problem:

$$
\left\{\begin{aligned}
-\operatorname{div} \sigma_{x}^{0}\left(d^{0}\right)-\operatorname{div}\left(\frac{1}{\left|Y_{S}\right|} \int_{Y_{S}} \sigma_{y}^{1}\left(d^{1}\right) d y\right) & =f_{S}, \quad \text { in } \Omega, \\
\sigma_{x}^{0}\left(d^{0}\right) n+\left(\frac{1}{\left|Y_{S}\right|} \int_{Y_{S}} \sigma_{y}^{1}\left(d^{1}\right) n d y\right) n & =0, \quad \text { on } \Gamma_{N}, \\
d^{0} & =0, \quad \text { on } \Gamma_{D},
\end{aligned}\right.
$$

and

$$
\left\{\begin{aligned}
\sigma_{y}\left(d^{1}\right) n+A\left(\int_{\partial Y_{F}} d^{1} \cdot n\right) n= & A\left|Y_{F}\right|\left(\operatorname{div}_{x} \sigma_{y} d^{0}\right) n \\
& -\sigma_{x}\left(d^{0}\right) n, \text { on } \Omega \times \partial Y_{F}, \\
& Y \text {-periodic. }
\end{aligned}\right.
$$

Remark 2.2 The case $A=0$ (that is, when there is no gas in the inclusions) corresponds to a periodic perforated elastic media with homogeneous Neumann boundary conditions on the holes, and we recover a more standard homogenized problem [10]. One of the difference here when $A \neq 0$, is that the boundary condition of the cell problem (57) is non standard due to the presence of the non local term. Moreover, and as it should be expected, the presence of gas in the holes changes the reaction to compression of the media (see the definition of $\sigma^{0}$ and $\sigma^{1}$ ).

\subsection{Homogenized problem}

In this subsection we rewrite (56), (57) uniquely in terms of $d^{0}$ and thus exhibit the homogenized stress tensor associated with the limit problem.

Using the superposition principle in (57) (or in the local problem $(L P)_{1}$ ), we have that

$$
d^{1}=\sum_{k, l=1}^{N}\left[e_{x}\left(d^{0}\right)\right]_{k l} \chi^{k l},
$$

where $\chi^{k l}, 1 \leq k, l \leq N$, is solution of

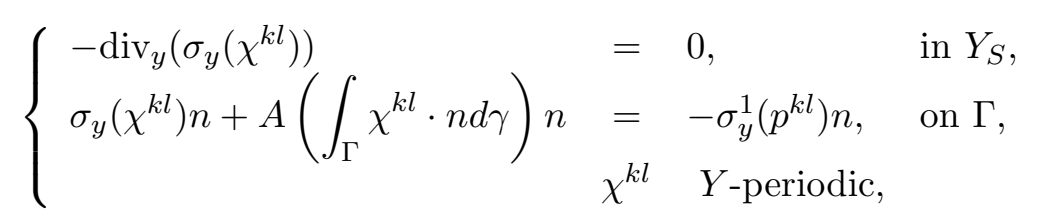

and where $p^{k l}$ is defined by

$$
p^{k l}(y)=\frac{1}{2}\left(y_{k} e^{l}+y_{l} e^{k}\right), 1 \leq k, l \leq N .
$$

Remark 2.3 Problem (59) is well-posed since it is easy to check that the compatibility condition (31) is satisfied. 
Remark 2.4 The stress tensors $\sigma, \sigma^{0}$ and $\sigma^{1}$, defined in (34)-(35), can be written in terms of a strain tensor e(.) and appropriately defined symmetric fourth-order tensors $\mathcal{A}, \mathcal{A}^{0}$ and $\mathcal{A}^{1}$. In fact, for $\sigma$ we have (see, for example, [31])

$$
\sigma(\cdot)=\mathcal{A} e(\cdot),
$$

with

$$
\mathcal{A}_{i j k l}=2 \mu \delta_{i j k l}+\lambda \delta_{i j} \delta_{k l}, \quad 1 \leq i, j, k, l \leq N
$$

and

$$
\delta_{i j k l}=\frac{1}{2}\left(\delta_{i k} \delta_{j l}+\delta_{i l} \delta_{j k}\right),
$$

( $\delta_{i j}$ stands for the Kronecker delta).

If $\mathcal{A}^{0}$ and $\mathcal{A}^{1}$ are defined in the same way as in (62) by replacing $\lambda$ by the modified Lamé constants (see (34)-(35)), $\lambda^{0}$ and $\lambda^{1}$, respectively, then the same relations are satisfied. for $d^{0}$

Replacing (58) in (36) -or (54)- and (55) and using (61) we obtain the homogenized problem

$$
\left\{\begin{aligned}
-\operatorname{div}_{x}\left(\mathcal{A}^{\text {hom }} e_{x}\left(d^{0}\right)\right) & =f, \text { in } \Omega \\
\mathcal{A}^{\text {hom }} e_{x}\left(d^{0}\right) n & =0, \text { on } \Gamma_{N}, \\
d^{0} & =0, \text { on } \Gamma_{D}
\end{aligned}\right.
$$

where $\mathcal{A}^{\text {hom }}$ is the fourth-order tensor given by

$$
\mathcal{A}_{i j k l}^{h o m}=\frac{1}{\left|Y_{S}\right|} \int_{Y_{S}}\left(\sigma\left(\phi^{k l}\right)_{i j}-A\left|Y_{F}\right| \operatorname{div} \chi^{k l} \delta_{i j}\right) d y+\frac{A\left|Y_{F}\right|^{2}}{\left|Y_{S}\right|} \delta_{i j} \delta_{k l},
$$

and $\phi^{k l}=p^{k l}+\chi^{k l}$.

The following proposition holds:

Proposition 2.6 The fourth-order tensor $\mathcal{A}^{\text {hom }}$ defined in (64) is such that

a) (symmetry) $\mathcal{A}_{i j k l}^{\text {hom }}=\mathcal{A}_{i j l k}^{\text {hom }}=\mathcal{A}_{\text {klij }}^{\text {hom }}$;

b) (ellipticity) there exists $\beta>0$ such that for any $N \times N$ symmetric matrix $\xi$

$$
\left(\mathcal{A}^{h o m} \xi\right): \xi \geq \beta \xi: \xi
$$

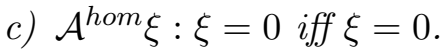

As a consequence of Proposition 2.6, that is proven in Appendix B, we have the following existence and uniqueness result:

Theorem 2.7 The homogenized problem (63) has a unique solution $d^{0} \in X_{D}$.

Proof. See for example [31].

Remark 2.5 The homogenized problem (63) obtained by two-scale convergence is exactly (34)(36) formally obtained with the asymptotic expansion. 


\section{Incompressible case: homogenization process}

\subsection{Incompressible elastic model}

We now study the case where the elastic media is assumed to be incompressible. The unknowns are now the displacement field $d^{\varepsilon}$, that satisfies the incompressibility constraint $\operatorname{div} d^{\varepsilon}=0$, and the pressure $q^{\varepsilon}$ which is the Lagrange multiplier associated with this constraint. The model now reads:

$$
\left\{\begin{aligned}
-\operatorname{div}\left(\sigma\left(d^{\varepsilon}, q^{\varepsilon}\right)\right) & =f_{S}, \quad \text { in } \Omega^{\varepsilon}, \\
\operatorname{div} d^{\varepsilon} & =0, \quad \text { in } \Omega^{\varepsilon}, \\
\sigma\left(d^{\varepsilon}, q^{\varepsilon}\right) n^{\varepsilon}+\frac{A}{\varepsilon^{N}}\left(\int_{Y^{\varepsilon, k}} d^{\varepsilon} \cdot n^{\varepsilon} d \gamma\right) n^{\varepsilon} & =0, \quad \text { on } \Gamma^{\varepsilon, k}, \quad \forall k \in \mathbb{Z}_{\varepsilon}^{N}, \\
\sigma\left(d^{\varepsilon}, q^{\varepsilon}\right) n & =0, \quad \text { on } \Gamma_{N}, \\
d^{\varepsilon} & =0, \quad \text { on } \Gamma_{D},
\end{aligned}\right.
$$

where we use the notations of previous sections (the constant $A$ was introduced in (12)) and tensor $\sigma\left(d^{\varepsilon}, q^{\varepsilon}\right)$ is defined by

$$
\sigma\left(d^{\varepsilon}, q^{\varepsilon}\right)=2 \mu e\left(d^{\varepsilon}\right)-q^{\varepsilon} I .
$$

In the next subsection, using the mixed variational formulation and a priori estimates, we will prove that problem (65) is well posed and that it can be rigorously obtained as the limit of (13) as $\lambda$ tends to infinity. Next, in order to obtain the limit of (65) as $\varepsilon$ goes to zero, we proceed as for the compressible case: first we explain what has to be changed in the two scale asymptotic expansion and then, we study the two scale limit. Finally we exhibit the homogenized problem.

\subsection{Mixed variational formulation}

Let $X_{D}^{\varepsilon}$ be the functional space introduced in section 2.1.1. Let $M^{\varepsilon}=L^{2}\left(\Omega^{\varepsilon}\right)$ be endowed with the norm $\|\cdot\|_{0, \Omega_{\varepsilon}}$. The mixed variational formulation of (65) is

$$
\left\{\begin{aligned}
\text { Find }\left(d^{\varepsilon}, p^{\varepsilon}\right) \in X_{D}^{\varepsilon} \times M^{\varepsilon} \text { such that } & \\
\left(a_{\mu}^{\varepsilon}\left(d^{\varepsilon}, \varphi^{\varepsilon}\right)+a_{\Gamma}^{\varepsilon}\left(d^{\varepsilon}, \varphi^{\varepsilon}\right)\right)+b^{\varepsilon}\left(\varphi^{\varepsilon}, q^{\varepsilon}\right) & =\ell^{\varepsilon}\left(\varphi^{\varepsilon}\right), \quad \forall \varphi^{\varepsilon} \in X_{D}^{\varepsilon}, \\
b^{\varepsilon}\left(d^{\varepsilon}, r^{\varepsilon}\right) & =0, \quad \forall r^{\varepsilon} \in M^{\varepsilon},
\end{aligned}\right.
$$

where $a_{\mu}^{\varepsilon}$ and $a_{\Gamma}^{\varepsilon}$ are the bilinear forms defined in (17), $\ell^{\varepsilon}$ is defined in (18), and $b^{\varepsilon}(\cdot, \cdot): X_{D}^{\varepsilon} \times M^{\varepsilon} \rightarrow$ $\mathbb{R}$ is the bilinear form defined by

$$
b^{\varepsilon}\left(\varphi^{\varepsilon}, r^{\varepsilon}\right)=-\int_{\Omega^{\varepsilon}} r^{\varepsilon} \operatorname{div} \varphi^{\varepsilon} d x .
$$

Since $a_{\mu}^{\varepsilon}+a_{\Gamma}^{\varepsilon}$ is elliptic and continuous in $X_{D}^{\varepsilon} \times X_{D}^{\varepsilon}$, in order to have existence and uniqueness for the mixed variational problem (66), the inf-sup condition has to be satisfied (see [16]), that is, we have to prove that there exists a constant $\beta>0$ such that

$$
\operatorname{rinf}_{r^{\varepsilon} \in M^{\varepsilon}} \sup _{\varphi^{\varepsilon} \in X_{D}^{\varepsilon}} \frac{b^{\varepsilon}\left(\varphi^{\varepsilon}, r^{\varepsilon}\right)}{\left.\left|\varphi^{\varepsilon}\right|_{1, \Omega^{\varepsilon}}|| r^{\varepsilon}\right|_{0, \Omega^{\varepsilon}}} \geq \beta .
$$

Proposition 3.1 The inf-sup condition holds uniformly in $\varepsilon$, i.e. there exists $\beta>0$, that does not depend on $\varepsilon$, such that (68) holds. 
Proof. Given $q^{\varepsilon} \in L^{2}\left(\Omega^{\varepsilon}\right)$, we extend it by (see [10])

$$
\widehat{q}^{\varepsilon}(x)= \begin{cases}q^{\varepsilon}(x) & x \in \Omega^{\varepsilon} \\ -\frac{1}{\left|\Omega \backslash \Omega^{\varepsilon}\right|} \int_{\Omega^{\varepsilon}} q^{\varepsilon}(x) d x, & x \in \Omega \backslash \Omega^{\varepsilon},\end{cases}
$$

and this extension is such that

$$
\left\|\widehat{q^{\varepsilon}}\right\|_{0, \Omega} \leq C_{1}\left\|q^{\varepsilon}\right\|_{0, \Omega^{\varepsilon}}
$$

with $C_{1}$ independent of $\varepsilon$ (see [10]). Moreover as $\widehat{q^{\varepsilon}}$ belongs to $L_{0}^{2}(\Omega)$, then there exists $\widehat{d} \in H_{0}^{1}(\Omega)$ (see [16]), such that

$$
\operatorname{div} \widehat{d}=\widehat{q^{\varepsilon}}, \quad \text { in } \Omega,
$$

and

$$
|\widehat{d}|_{1, \Omega} \leq\left. C_{2}|| \widehat{q^{\varepsilon}}\right|_{0, \Omega},
$$

with $C_{2}$ also independent of $\varepsilon$. Let $z^{\varepsilon}$ be the restriction of $\widehat{d}$ to $\Omega^{\varepsilon}$. Then $z^{\varepsilon}$ belongs to $X_{D}^{\varepsilon}$ (in fact, it is equal to 0 on the whole boundary $\partial \Omega$, not only on $\Gamma_{D}$ ), and is such that

$$
\operatorname{div} z^{\varepsilon}=q^{\varepsilon}, \text { in } \Omega^{\varepsilon}
$$

and

$$
\left|z^{\varepsilon}\right|_{1, \Omega^{\varepsilon}} \leq|\widehat{d}|_{1, \Omega} \leq C_{2}|| \widehat{q^{\varepsilon}}\left\|_{0, \Omega} \leq C_{1} C_{2}\right\| q^{\varepsilon} \|_{0, \Omega^{\varepsilon}} .
$$

Now, using (71) and (72) we have that

$$
\begin{aligned}
\int_{\Omega^{\varepsilon}} q^{\varepsilon} \operatorname{div} z^{\varepsilon} d x & =\int_{\Omega^{\varepsilon}}\left|q^{\varepsilon}\right|^{2} d x \\
& \geq\left.\left(C_{1} C_{2}\right)^{-1}|| q^{\varepsilon}\right|_{0, \Omega^{\varepsilon}}\left|z^{\varepsilon}\right|_{1, \Omega^{\varepsilon}} .
\end{aligned}
$$

Hence the inf-sup condition holds with a constant $\beta=\left(C_{1} C_{2}\right)^{-1}$ that does not depend on $\varepsilon$.

With the ellipticity and continuity of $a_{\mu}^{\varepsilon}+a_{\Gamma}^{\varepsilon}$ and the inf-sup condition we have the following existence and uniqueness result:

Theorem 3.2 Given $f_{S} \in L^{2}(\Omega)$, there exists a unique solution $\left(d^{\varepsilon}, q^{\varepsilon}\right)$ to the mixed variational problem (66) in $X_{D}^{\varepsilon} \times M^{\varepsilon}$.

Remark 3.1 It is not difficult to see that $d^{\varepsilon}$ can be obtained as the limit of $\left\{d_{\lambda}^{\varepsilon}\right\}_{\lambda \geq 0}$ solutions of (13) as $\lambda$ goes to infinity. Moreover it is easy to check that $q^{\varepsilon}$ is the weak limit in $L^{2}\left(\Omega^{\varepsilon}\right)$ of $q_{\lambda}^{\varepsilon}=\lambda \operatorname{div}\left(d_{\lambda}^{\varepsilon}\right)$.

\subsection{A priori estimates}

In this section we get uniform a priori estimates for the extension of $\left(d^{\varepsilon}, q^{\varepsilon}\right)$ solution of $(66)$. We have

Proposition 3.3 Let $f_{S} \in L^{2}(\Omega)$. If $\left(d^{\varepsilon}, p^{\varepsilon}\right) \in X_{D}^{\varepsilon} \times M^{\varepsilon}$ is the weak solution of (14) (i.e. solution of the mixed variational problem (66)), then

$$
\begin{aligned}
\left|\widetilde{d^{\varepsilon}}\right|_{1, \Omega} & \leq C\left\|f_{S}\right\|_{0, \Omega}, \\
\|\left.\widehat{q^{\varepsilon}}\right|_{0, \Omega} & \leq C\left\|f_{S}\right\|_{0, \Omega},
\end{aligned}
$$

where ${ }^{\sim}$ and ${ }^{\wedge}$ denote the extension operators introduced in (19) and (69), respectively, and $C$ stands for constants independent of $\varepsilon$.

Proof. The proof relies on standard arguments. Indeed, using properties of the extension operator , and Korn's and Poincaré's inequalities in $X_{D}^{\varepsilon}$ and $X_{D}$ lead to (74). Moreover, from the inf-sup condition we can get uniform estimates for $\widehat{q^{\varepsilon}}$ once we have uniform estimates for $\widetilde{d}^{\varepsilon}$. 


\subsection{Two-scale asymptotic expansion method}

As for the "compressible" case we can obtain the homogenized problem by a two scale asymptotic expansion. The linear model (14) proposed for the incompressible elastic material with gaseous inclusions is similar to the problem studied in [10] in the case of a fluid in a periodically perforated domain with Neumann or Fourier boundary conditions on the inclusion's boundaries.

As in [10], we propose the following ansatz for $d^{\varepsilon}$ and $q^{\varepsilon}$ :

$$
\begin{aligned}
& d^{\varepsilon}(x)=d^{0}(x, x / \varepsilon)+\varepsilon d^{1}(x, x / \varepsilon)+\varepsilon^{2} d^{2}(x, x / \varepsilon)+\ldots, \\
& q^{\varepsilon}(x)=\frac{1}{\varepsilon} q^{-1}(x, x / \varepsilon)+q^{0}(x, x / \varepsilon)+\varepsilon q^{1}(x, x / \varepsilon)+\ldots,
\end{aligned}
$$

where $d^{k}$ and $q^{k}$ are functions such that $d^{k}: \Omega \times Y \rightarrow \mathbb{R}^{N}, q^{k}: \Omega \times Y \rightarrow \mathbb{R}$ and $Y$-periodic in the rapid variable $y$.

The main difference between the incompressible case and the previous one is the constraint $\operatorname{div} d^{\varepsilon}=0$, which implies the following relations

$$
\operatorname{div}_{y} d^{0}=0 \text { in } Y_{s} \text { and } \operatorname{div}_{x} d^{k-1}+\operatorname{div}_{y} d^{k}=0 \text { in } Y_{s},
$$

for every $k \geq 1$.

Replacing these expansions in (14) and by comparison of powers of $\varepsilon$ we find local problems that we will denote by $(L P)_{k}^{\text {inc }}(k=0,1,2, \ldots)$, which are the analogous of local problems $(L P)_{k}$ obtained by replacing $\sigma_{y}\left(d^{k}\right)$ with $\sigma_{y}\left(d^{k}, q^{k-1}\right)$ and $\sigma_{x}\left(d^{k}\right)$ with $\sigma_{x}\left(d^{k}, q^{k-1}\right)$, for $k=0,1, \ldots$ and by adding the corresponding local divergence constraint (76).

Combining these relations, the first local problem is:

$$
(L P)_{0}^{i n c} \mid \begin{aligned}
-\operatorname{div}_{y} \sigma_{y}\left(d^{0}, q^{-1}\right) & = & 0, & \\
\operatorname{div}_{y} d^{0}= & 0, & & \text { in } Y_{S}, \\
\sigma_{y}\left(d^{0}, q^{-1}\right) n & = & 0, & \text { on } \Gamma, \\
q^{-1}, d^{0} & & Y \text {-periodic, } &
\end{aligned}
$$

whose unique solution is the trivial solution $\left(d^{0}, q^{-1}\right)=\left(d^{0}(x), 0\right)$ (that is, $d^{0}$ equal to 0 up to an additive constant in $y$ ).

In order to simplify the next local problems we remark that the constraints (76) can be used to rewrite the surface integral $A \int_{\Gamma} d^{k} \cdot n$ that appears in the left-hand side of the boundary condition on $\Gamma$ in each local problems $(L P)_{k}^{i n c}$. Indeed, remembering that $d^{k}$ is periodic with respect to $y$

$$
\int_{\Gamma} d^{0} \cdot n=\int_{Y_{S}} \operatorname{div}_{y} d^{0} d y=0
$$

and for $k \geq 1$, we have the following recursion formula for the unknown surface integral terms

$$
\int_{\Gamma} d^{k} \cdot n=\int_{Y_{S}} \operatorname{div}_{y} d^{k} d y=-\int_{Y_{S}} \operatorname{div}_{x} d^{k-1} d y .
$$

Finally, the relations in (76) and the fact that $d^{0}$ depends only on $x$ are also used to expand other terms that appear in the boundary condition on $\Gamma$ as, for example, the terms

$$
\begin{aligned}
\int_{Y_{F}} \operatorname{div}_{y} d^{1}\left(x, y^{\prime}\right) d y^{\prime}=-\int_{\Gamma} d^{1} \cdot n & =-\int_{Y_{S}} \operatorname{div}_{y} d^{1}\left(x, y^{\prime}\right) d y^{\prime} \\
& =\left|Y_{S}\right| \operatorname{div}_{x} d^{0}(x),
\end{aligned}
$$


and using this equality

$$
\begin{aligned}
\int_{Y_{F}} y \cdot \nabla_{x}\left(\operatorname{div}_{x} d^{0}(x)+\operatorname{div}_{y} d^{1}\left(x, y^{\prime}\right)\right) d y^{\prime} & =\left|Y_{F}\right| y \cdot \nabla_{x} \operatorname{div}_{x} d^{0}+y \cdot \nabla_{x} \int_{Y_{F}} \operatorname{div}_{x} d^{1}\left(x, y^{\prime}\right) d y^{\prime} \\
& =\left|Y_{F}\right| y \cdot \nabla_{x} \operatorname{div}_{x} d^{0}+\left|Y_{S}\right| y \cdot \nabla_{x} \operatorname{div}_{x} d^{0} \\
& =|Y| y \cdot \nabla_{x} \operatorname{div}_{x} d^{0}
\end{aligned}
$$

Consequently we have

$$
\begin{aligned}
& (L P)_{1}^{i n c} \mid-\operatorname{div}_{y} \sigma_{y}\left(d^{1}, q^{0}\right)=0, \quad \text { in } Y_{S}, \\
& \operatorname{div}_{y} d^{1}=-\operatorname{div}_{x} d^{0}, \quad \text { in } Y_{S}, \\
& \sigma_{y}\left(d^{1}, q^{0}\right) n=-\sigma_{x}\left(d^{0}, 0\right) n+A\left(|Y| \operatorname{div}_{x} d^{0}\right) n, \quad \text { on } \Gamma, \\
& q^{0}, d^{1} \quad Y \text {-periodic, }
\end{aligned}
$$

and

$$
(L P)_{2}^{i n c} \mid \begin{array}{rlrl}
-\operatorname{div}_{y} \sigma_{y}\left(d^{2}, q^{1}\right)= & \operatorname{div}_{x} \sigma_{y}\left(d^{1}, q^{0}\right)+\operatorname{div}_{x} \sigma_{x}\left(d^{0}, 0\right) & \\
& +\operatorname{div}_{y} \sigma_{x}\left(d^{1}, 0\right)+f_{S}, & \\
\operatorname{div}_{y} d^{2}= & -\operatorname{div}_{x} d^{1}, & \text { in } Y_{S}, \\
\sigma_{y}\left(d^{2}, q^{1}\right) n= & -\sigma_{x}\left(d^{1}, 0\right) n \\
& +A\left(\int_{Y} \operatorname{div}_{x} d^{1} d y^{\prime}-|Y| y \cdot \nabla_{x} \operatorname{div}_{x} d^{0}\right) n & \\
& +A\left(\int_{Y_{F}} y^{\prime} \cdot \nabla_{x}\left(\operatorname{div}_{x} d^{0}+\operatorname{div}_{y} d^{1}\right) d y^{\prime}\right) n, & & \\
& & & \\
& & &
\end{array}
$$

Remark 3.2 We can note here that, due to the incompressibility constraint the non standard non local boundary conditions are replaced, in the local problems, by standard Neumann boundary conditions.

In order to have existence and uniqueness of solutions for problems $(L P)_{k}^{i n c}(k=1,2)$ a compatibility condition must be satisfied:

$$
\int_{Y_{S}} F_{k} d y+\int_{\Gamma} G_{k}=0, \quad k=1,2
$$

where $F_{k}$ and $G_{k}$ stand, respectively, for the right-hand side in the momentum equation and the right-hand side in the boundary condition on $\Gamma$ in $(L P)_{k}^{i n c}$. It is easy to see that the compatibility condition for $(L P)_{1}^{i n c}$ is satisfied. Using Green's formula in the compatibility condition for $(L P)_{2}^{i n c}$, we arrive to the following equation for $d^{0}$ :

$$
\begin{aligned}
-\operatorname{div}_{x}\left(2 \mu e_{x}\left(d^{0}\right)\right)= & \frac{1}{\left|Y_{S}\right|} \int_{Y_{S}} f_{S}+\operatorname{div}_{x}\left(\frac{1}{\left|Y_{S}\right|} \int_{Y_{S}} \sigma_{y}\left(d^{1}, q^{0}\right)\right) \\
& -A \frac{|Y|}{\left|Y_{S}\right|}\left(\int_{\Gamma} n \otimes y\right) \nabla_{x} \operatorname{div}_{x} d^{0},
\end{aligned}
$$


where we have used that $\int_{\Gamma} g(x) n=0$ and the notation $(a \otimes b)$ introduced in (33). Furthermore we have that

$$
-A \frac{|Y|}{\left|Y_{S}\right|}\left(\int_{\Gamma} n \otimes y d \gamma\right)=A \frac{|Y|\left|Y_{F}\right|}{\left|Y_{S}\right|} I
$$

thus

$$
\begin{aligned}
-\operatorname{div}_{x}\left(2 \mu e_{x}\left(d^{0}\right)\right)= & \frac{1}{\left|Y_{S}\right|} \int_{Y_{S}} f_{S}+\operatorname{div}_{x}\left(\frac{1}{\left|Y_{S}\right|} \int_{Y_{S}} \sigma_{y}\left(d^{1}, q^{0}\right)\right) \\
& +A \frac{|Y|\left|Y_{F}\right|}{\left|Y_{S}\right|} \nabla_{x} \operatorname{div}_{x} d^{0} .
\end{aligned}
$$

Remark 3.3 Note that as expected, since the gas is compressible, the limit problem satisfied by $d^{0}$ is also "compressible".

Remark 3.4 For $A=0$ we recover standard results for the homogenized Stokes equations in perforated domain with Neumann boundary conditions.

\subsection{Two-scale convergence method}

In this section we use the two-scale convergence method in order to obtain the two-scale homogenized problem associated to (14).

As in section 2.3 we use the a-priori estimates of Proposition 3.3 to prove the following

Proposition 3.4 Let $\left(\widetilde{d^{\varepsilon}}, \widehat{q^{\varepsilon}}\right)$ be the sequence of extended solution of mixed variational problem (66). Then there exist $q^{0} \in L^{2}\left(\Omega ; L_{\#}^{2}(Y)\right), d^{0} \in X_{D}$ and $d^{1} \in L^{2}\left(\Omega ; H_{\#}^{1}(Y) / \mathbb{R}\right)^{N}$ such that, as $\varepsilon$ tends to 0 ,

$$
\begin{aligned}
& \widetilde{d}^{\varepsilon} \stackrel{2}{\rightarrow} d^{0}, \\
& \nabla \widetilde{d^{\varepsilon}} \stackrel{2}{\rightarrow} \nabla_{x} d^{0}+\nabla_{y} d^{1}, \\
& \operatorname{div} \widetilde{d^{\varepsilon}} \stackrel{2}{\rightarrow} \operatorname{div}_{x} d^{0}+\operatorname{div}_{y} d^{1}, \\
& e\left(\widetilde{d^{\varepsilon}}\right) \stackrel{2}{\rightarrow} e_{x}\left(d^{0}\right)+e_{y}\left(d^{1}\right), \\
& \widehat{q}^{\varepsilon} \stackrel{2}{\rightarrow} q^{0} .
\end{aligned}
$$

With this proposition and Lemma 2.3 we are able to prove, as in section 2.3 , the following

Theorem 3.5 Let $\left(d^{\varepsilon}, q^{\varepsilon}\right)_{\varepsilon>0}$ be the sequence of weak solution of $(66)$. Then the sequence $\left(\widetilde{d^{\varepsilon}}, \widehat{q^{\varepsilon}}\right)_{\varepsilon>0}$ two-scale converges to $\left(d^{0}, q^{0}\right) \in X_{D} \times L^{2}\left(\Omega ; L_{\#}^{2}(Y)\right)$ and $\nabla \widetilde{d^{\varepsilon}}$ two scale converges to $\nabla_{x} d^{0}+\nabla_{y} d^{1}$ where $\left(d^{0}, d^{1} \chi_{S}, q^{0} \chi_{S}\right) \in X_{D} \times L^{2}\left(\Omega ; H_{\#}^{1}\left(Y_{S}\right) / \mathbb{R}\right)^{N} \times L^{2}\left(\Omega ; L_{\#}^{2}(Y)\right)$ is the unique solution of the following variational two-scale homogenized problem

$$
\begin{aligned}
2 \mu \int_{\Omega} \int_{Y_{S}}\left(e_{x}\left(d^{0}\right)+e_{y}\left(d^{1}\right)\right):\left(e_{x}(\phi)+e_{y}\left(\phi^{1}\right)\right) d y d x & \\
+A \int_{\Omega}\left(\left|Y_{F}\right| \operatorname{div}_{x} d^{0}+\int_{\Gamma} d^{1} \cdot n\right)\left(\left|Y_{F}\right| \operatorname{div}_{x} \phi+\int_{\Gamma} \phi^{1} \cdot n\right) d x & \\
-\int_{\Omega} \int_{Y_{S}} q^{0}\left(\operatorname{div}_{x} \phi+\operatorname{div}_{y} \phi^{1}\right) d y d x & =\left|Y_{S}\right| \int_{\Omega} f_{S} \cdot \phi d x, \\
\int_{\Omega} \int_{Y_{S}}\left(\operatorname{div}_{x} d^{0}+\operatorname{div}_{y} d^{1}\right) \eta d y d x & =0,
\end{aligned}
$$


for all $\left(\phi, \phi^{1}, \eta\right)$ in $X_{D} \times L^{2}\left(\Omega ; H_{\#}^{1}\left(Y_{S}\right) / \mathbb{R}\right)^{N} \times L^{2}\left(\Omega ; L_{\#}^{2}\left(Y_{S}\right)\right)$.

Proof. The theorem can be proven exactly as Theorem 2.4, the only point to verify is the existence and uniqueness of the limit pressure $q^{0}$. This can be done by proving the following two scale inf-sup condition:

Lemma 3.6 There exists a constant $\beta>0$ such that for all $p \in L^{2}\left(\Omega ; L_{\#}^{2}\left(Y_{S}\right)\right)$

$$
\sup _{\left(\phi, \phi^{1}\right) \in X_{D} \times L^{2}\left(\Omega ; H_{\#}^{1}\left(Y_{S}\right) / \mathbb{R}\right)^{N}} \frac{\int_{\Omega} \int_{Y_{S}} p\left(\operatorname{div}_{x} \phi+\operatorname{div}_{y} \phi^{1}\right)}{\left\|\phi, \phi^{1}\right\|_{X_{D} \times L^{2}\left(\Omega ; H_{\#}^{1}\left(Y_{S}\right) / \mathbb{R}\right)^{N}}} \geq \beta\|p\|_{L^{2}\left(\Omega ; L_{\#}^{2}\left(Y_{S}\right)\right)} .
$$

This lemma, which is proven in Appendix $\mathrm{C}$, is equivalent to the fact that the mapping $T$ defined by

$$
\begin{aligned}
X_{D} \times L^{2}\left(\Omega ; H_{\#}^{1}\left(Y_{S}\right) / \mathbb{R}\right)^{N} & \rightarrow L^{2}\left(\Omega ; L_{\#}^{2}\left(Y_{S}\right)\right) \\
\left(\phi, \phi^{1}\right) & \mapsto \operatorname{div}_{x} \phi+\operatorname{div}_{y} \phi^{1}
\end{aligned}
$$

is an isomorphism on ker $T^{\perp}$.

Remark 3.5 By proceeding exactly as for (14), it is an easy task to verify that the two-scale problem (83) can be obtained from (46) by letting $\lambda$ go to infinity. Consequently we have the following commutative diagram

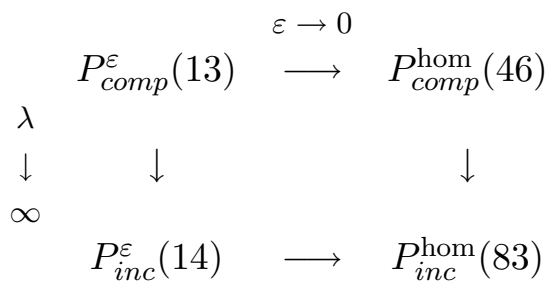

As in the compressible case, using appropriate test functions $\phi$ and $\phi^{1}$, we can find the corresponding strong two-scale boundary-value problem associated to the above two-scale variational problem (83):

$$
\left\{\begin{aligned}
-\operatorname{div}\left(2 \mu e_{x}\left(d^{0}\right)+\frac{1}{\left|Y_{S}\right|} \int_{Y_{S}} \sigma_{y}\left(d^{1}, q^{0}\right)+A \frac{|Y|\left|Y_{F}\right|}{\left|Y_{S}\right|} \operatorname{div}_{x} d^{0}\right) & =f_{S}, & & \text { in } \Omega, \\
\left(2 \mu e_{x}\left(d^{0}\right)+\frac{1}{\left|Y_{S}\right|} \int_{Y_{S}} \sigma_{y}\left(d^{1}, q^{0}\right)+A \frac{|Y|\left|Y_{F}\right|}{\left|Y_{S}\right|} \operatorname{div}_{x} d^{0}\right) n & =0, & & \text { on } \Gamma_{N}, \\
d^{0} & =0, & & \text { on } \Gamma_{D},
\end{aligned}\right.
$$

where the cell problem reads

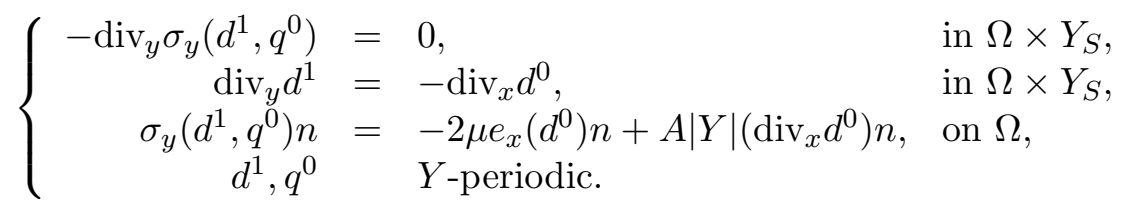




\subsection{Homogenized equation}

In order to obtain the homogenized equation and in particular the homogenized stress tensor, we introduce the following auxiliary local problems:

$$
(L P)_{k l}^{i n c} \mid \begin{aligned}
-\operatorname{div}_{y}\left(\sigma_{y}\left(\chi^{k l}, \eta^{k l}\right)\right) & =0, & & \text { in } Y_{S}, \\
\operatorname{div}_{y} \chi^{k l} & =-\delta_{k l}, & & \text { in } Y_{S}, \\
\sigma_{y}\left(\chi^{k l}, \eta^{k l}\right) n & =-\mathcal{A}^{l o c} e_{y}\left(p^{k l}\right) n, & & \text { on } \Gamma, \\
\eta^{k l}, \chi^{k l} Y \text {-periodic, } & & &
\end{aligned}
$$

where $\mathcal{A}^{l o c}$ is the fourth-order tensor defined by

$$
\mathcal{A}_{i j k l}^{l o c}=2 \mu \delta_{i j k l}-A|Y| \delta_{i j} \delta_{k l},
$$

and $p^{k l}$ is the polynomial defined in (60). It follows that

$$
\mathcal{A}^{l o c} e_{y}\left(p^{k l}\right)=2 \mu e_{y}\left(p^{k l}\right)-A|Y| \operatorname{div}_{y} p^{k l} I .
$$

Therefore, by superposition, the solution of $(L P)_{1}^{i n c}$ can be written as

$$
d^{1}=\sum_{k, l=1}^{N}\left[e_{x}\left(d^{0}\right)\right]_{k l} \chi^{k l}, \quad q^{0}=\sum_{k, l=1}^{N}\left[e_{x}\left(d^{0}\right)\right]_{k l} \eta^{k l} .
$$

Let us define the fourth order tensor $\mathcal{A}_{\text {inc }}^{\text {hom }}$ by

$$
\left(\mathcal{A}_{i n c}^{\text {hom }}\right)_{i j k l}=2 \mu \delta_{i j k l}+\frac{1}{\left|Y_{S}\right|} \int_{Y_{S}}\left[\sigma_{y}\left(\chi^{k l}, \eta^{k l}\right)\right]_{i j} d y
$$

and the matrix $B^{\text {hom }}$ by

$$
\begin{aligned}
B^{\text {hom }} & =A|Y| \frac{\left|Y_{F}\right|}{\left|Y_{S}\right|} I \\
& =\frac{p_{a}|Y|}{N\left|Y_{S}\right|} I .
\end{aligned}
$$

Therefore the homogenized equation is

$$
-\operatorname{div}_{x}\left(\mathcal{A}_{i n c}^{\text {hom }} e_{x}\left(d^{0}\right)+B^{h o m} \operatorname{div}_{x} d^{0}\right)=f_{S} .
$$

Adding the associated Dirichlet and Neumann boundary condition on $\Gamma_{D}$ and $\Gamma_{N}$, respectively, we obtain the following limit equation

$$
\left\{\begin{aligned}
-\operatorname{div}_{x}\left(\mathcal{A}_{i n c}^{\text {hom }} e_{x}\left(d^{0}\right)+B^{\text {hom }} \operatorname{div}_{x} d^{0}\right) & =f_{S}, \text { in } \Omega \\
\left(\mathcal{A}_{\text {inc }}^{\text {hom }} e_{x}\left(d^{0}\right)+B^{\text {hom }} \operatorname{div}_{x} d^{0}\right) n & =0, \text { on } \Gamma_{N}, \\
d^{0} & =0, \text { on } \Gamma_{D} .
\end{aligned}\right.
$$

The following proposition holds

Proposition 3.7 The fourth order tensor $\mathcal{A}_{\text {inc }}^{\text {hom }}$ defined in (85) satisfies the following properties: 
a) (symmetry) $\left(\mathcal{A}_{\text {inc }}^{\text {hom }}\right)_{i j k l}=\left(\left(\mathcal{A}_{\text {inc }}^{\text {hom }}\right)_{k l i j}=\left(\mathcal{A}_{\text {inc }}^{\text {hom }}\right)_{i j l k}\right.$;

b) (ellipticity) there exists $\beta>0$ such that for any $N \times N$ symmetric matrix $\xi$

$$
\left(\mathcal{A}_{\text {inc }}^{\text {hom }} \xi\right): \xi \geq \beta \xi: \xi
$$

c) $\mathcal{A}_{\text {inc }}^{\text {hom }} \xi=0$ iff $\xi=0$.

Proof. The proof is similar as in proposition 2.6. The main idea is to prove the following identity

$$
\left(\mathcal{A}_{i n c}^{h o m}\right)_{i j k l}=a_{y}\left(\phi^{k l}, \phi^{i j}\right)+A|Y| \delta_{i j} \delta_{k l},
$$

where $a_{y}(\cdot, \cdot)$ is the bilinear form from $H_{\#}^{1}\left(Y_{S}\right) \times H_{\#}^{1}\left(Y_{S}\right)$ in $\mathbb{R}$ defined by

$$
a_{y}(\chi, \varphi)=\frac{2 \mu}{\left|Y_{S}\right|} \int_{Y_{S}} e_{y}(\chi): e_{y}(\varphi) d y
$$

Remark 3.6 The homogenized problem (87) obtained by two-scale convergence is exactly (77) formally obtained with the asymptotic expansion.

\section{Numerical calculations of the homogenized coefficients, incom- pressible case}

In this section we compute numerically the homogenized coefficients defined in (85) and (86).

First, we compute the entries of the tensor $\mathcal{A}_{\text {inc }}^{\text {hom }}$ (see (85)) for different incompressible elastic materials, which are determined by the Lamé constant $\mu$, for different ratios $\left|Y_{F}\right| /\left|Y_{S}\right|$, and for the cases $p_{a}=0$ and $p_{a}=10^{5} \mathrm{~Pa}$. That is, we compare the homogenized coefficients in the case of void inclusions with the case of inclusions filled with a gas at atmospheric pressure. The results show that the difference between the homogenized coefficients in the case of void inclusions and gas inclusions is more important when a softer materials (like living tissues) is considered, and it can be neglected in the case of a stiffer material (like rubber). Also, the influence of the cell's geometry can be observed.

Finally, we evaluate the entries of $B^{\text {hom }}$ (see (86)) for the same cell's geometries used in the calculation of $\mathcal{A}_{\text {inc }}^{\text {hom }}$ and for $p_{a}=10^{5}$.

\section{1 $\mathcal{A}_{\text {inc }}^{\text {hom }}$ tensor, approximation of local problems}

In order to compute the coefficients of the homogenized tensor $\mathcal{A}_{\text {inc }}^{\text {hom }}$ defined in (85) (and that, in what follows, we will simply denote by $a_{i j k l}$ ) we solve numerically $(L P)_{k l}^{i n c}$ (that we will denote by $\left.P_{k l}\right)$. We use a stable mixed finite element approximation $(P 1 i s o P 2 / P 1)$. The periodic boundary conditions are incorporated to the FEM spaces by adding the corresponding basis functions associated with the left-right and up-down boundary nodes and by adding the four basis functions associated with the corner's nodes. Note that $\chi^{k l} \in\left(H_{\#}^{1}\left(Y_{S}\right) / \mathbb{R}\right)^{N}$, therefore in the corresponding FEM space, the basis function associated to the remaining corner node is eliminated.

Following the symmetry property of proposition 3.7, from the 16 entries of tensor $\mathcal{A}_{\text {inc }}^{\text {hom }}$ there are only six different values. Therefore, we define $a_{1}=a_{1111}, a_{2}=a_{1212}\left(=a_{1221}=a_{2112}=a_{2121}\right)$, $a_{3}=a_{1122}\left(=a_{2211}\right), a_{4}=a_{1121}\left(=a_{1211}=a_{2111}=a_{1112}\right), a_{5}=a_{1222}\left(=a_{2122}=a_{2212}=a_{2221}\right)$ and $a_{6}=a_{2222}$. 
Moreover, following [11], if the domain $Y_{F}$ presents symmetry with respect to axis $O X$ and $O Y$, then from the above six values in $\mathcal{A}_{\text {inc }}^{\text {hom }}$ we have only three different values, since $a_{1}=a_{6}$ and $a_{4}=a_{5}=0$.

Remark 4.1 With these properties we see that we only need to solve two local problems, for example $P_{11}$ (to calculate $a_{1}$ and $a_{3}$ ) and $P_{12}$ (to calculate $a_{2}$ ), instead of the original three.

In order to avoid corner singularities we use $Y_{F}=\left\{(x, y) \in \mathbb{R}^{2}:(x-1 / 2)^{2}+(y-1 / 2)^{2}<r_{F}^{2}\right\}$ with $0<r_{F}<1 / 2$ (see Figure 2).
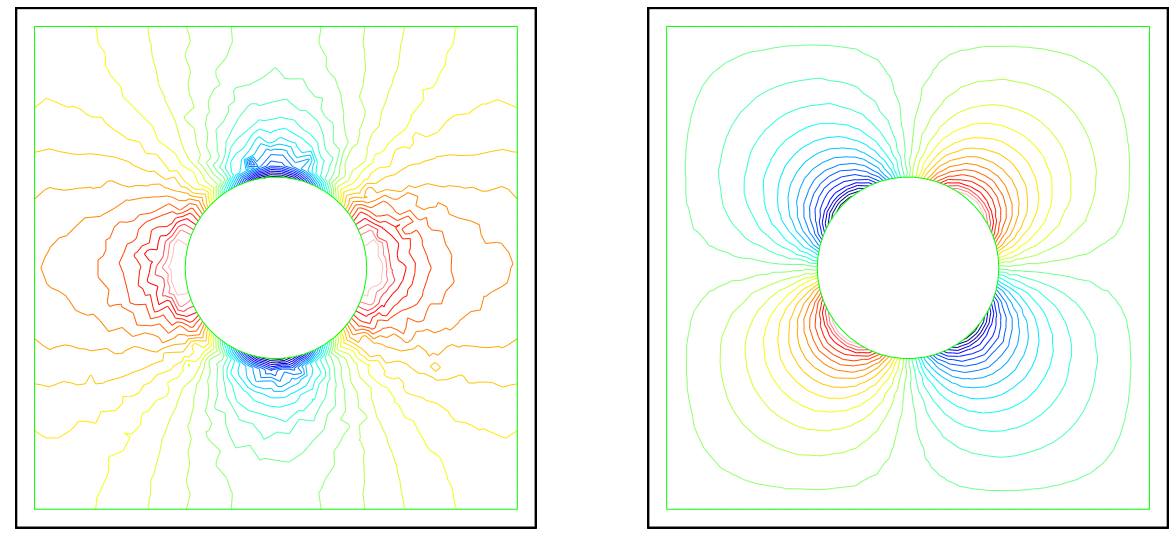

Figure 2: $\eta^{k l}$ pressure iso-values, case $k l=11$ (left) and $k l=12$ (right).

We consider three different values for the Lamé constant $\mu: \mu=10^{5} \mathrm{~Pa}, 10^{6} \mathrm{~Pa}$ and $10^{9} \mathrm{~Pa}$. The first two values correspond to the case of a living tissue material (see [29]), and the last one, to the case of an incompressible rubbery material. The results show that in the case of a soft elastic material (such as living tissues), the absence or presence of gas in the bubbles produces significant variations in the homogenized coefficients.

The graphics in Figure 3 present the calculated values of $a_{1}, a_{2}$ and $a_{3}$ as functions of $d_{F}=2 r_{F}$ . Each figure corresponds to a different value of $\mu$, and in each of them there are displayed the curves for the cases $p_{a}=0$ and $p_{a}=10^{5}$.

Remark 4.2 From Figure 3 we can observe that the presence of gas rigidifies the homogenized material (since $\left.a_{i}\left(p_{a}=0\right)<a_{i}\left(p_{a}=10^{5}\right), i=1,3\right)$. We can also observe that the smaller the inclusion is (i.e. $d_{F} \rightarrow 0$ ), the stiffer the homogenized material results, or inversely, the greater the inclusion is (i.e. $\left.d_{F} \rightarrow 1\right)$ the softer the homogenized material results. Finally, as expected by the definition of $P_{12}$, no difference is observed for $a_{2}$.

Remark 4.3 In the human parenchyma geometry, we have that the alveola's diameter $d_{\text {alv }}$ could vary between $75 \mu \mathrm{m}$ and $360 \mu \mathrm{m}$, and the thickness of the alveolar walls $h_{\text {wall }}$ is approximately $5 \mu \mathrm{m}$ (see [20]). Therefore the ratio $\rho=h_{\text {wall }} / d_{\text {alv }}$ vary between $1 / 72$ and $1 / 15$. By applying these proportions to our cell's geometry, we have that $d_{F}$ will vary from $15 / 17 \approx 0.8824$ to $d_{F}=72 / 74 \approx 0.9730$ (where we have used $h_{\text {wall }}=\rho(\Gamma, \partial Y)$ ). Hence, in view of an application to human physiology we should use $d_{F} \approx 0.9$, or even $d_{F} \approx 1$, however, having it close to 1 would lead to the resolution of local problems in very thin domains, with the corresponding numerical difficulties that situation like this implies. 

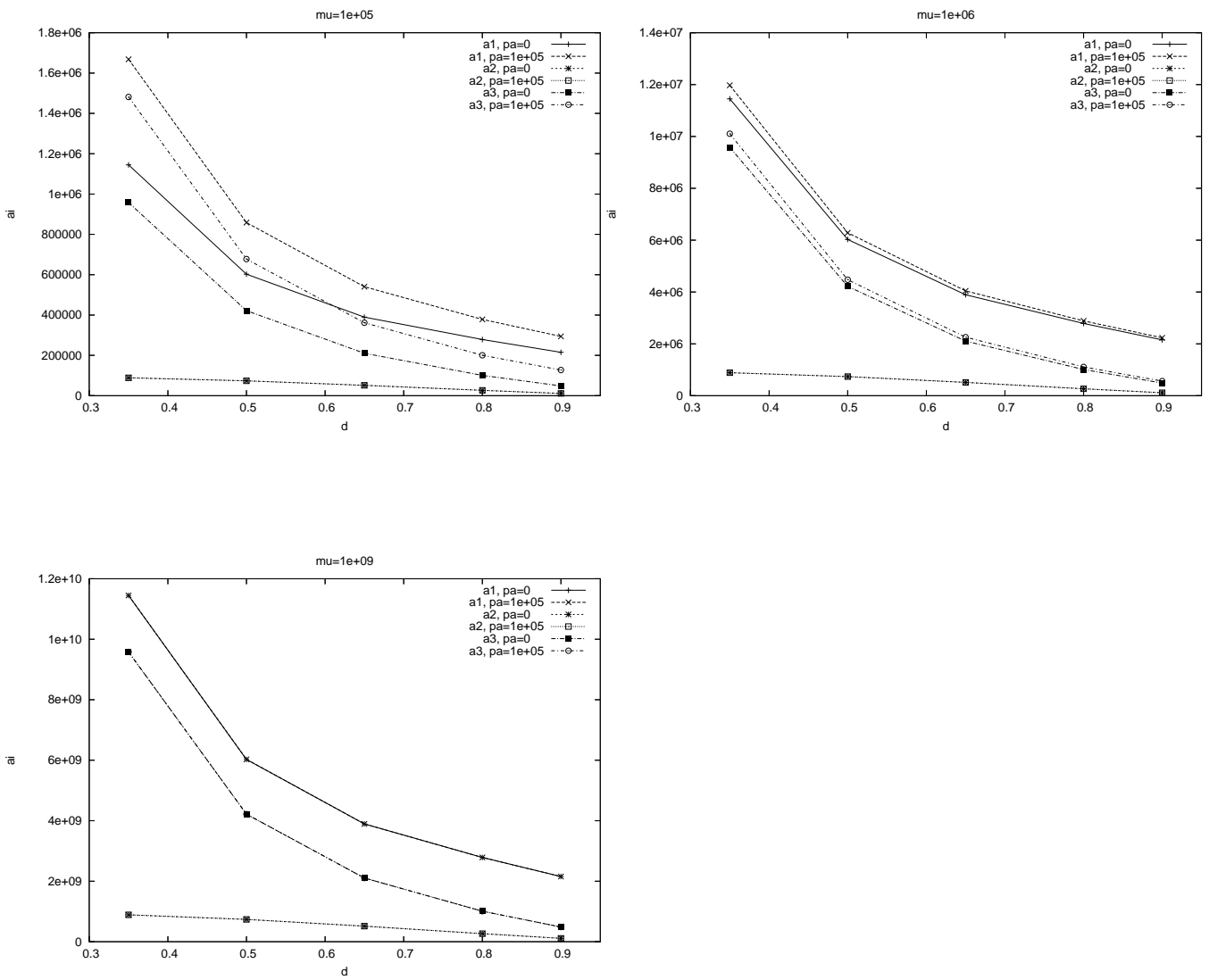

Figure 3: $a_{1}(+$ and $\times), a_{2}(*$ and $\square)$, and $a_{3}(\mathbf{\square}$ and $\circ),\left(+, *, \mathbf{\square}: p_{a}=0, \times, \square, \circ: p_{a}=10^{5} P a\right.$ ), for different diameters, for $\mu=10^{5} \mathrm{~Pa}$ (top-left), $\mu=10^{6} \mathrm{~Pa}$ (top-right) and $\mu=10^{9} \mathrm{~Pa}$ (bottom-left).

\section{2 $B^{\text {hom }}$ matrix}

Let us define $b_{\text {hom }}=\frac{p_{a}|Y|}{N\left|Y_{S}\right|}$, therefore, $B^{\text {hom }}=b_{\text {hom }} I$ (see (86)). Figure 4 presents the calculated values of $b_{\text {hom }}$ for $p_{a}=10^{5} \mathrm{~Pa}, N=2,|Y|=1$, and $\left|Y_{S}\right|=1-\pi r_{F}^{2}$.

Remark 4.4 From the definition of $b_{\text {hom }}$ we see that if $d_{F}$ tends to 1 , then $b_{\text {hom }}$ tends to $+\infty$. On the other hand, if $d_{F}$ tends to 0 , then $b_{\text {hom }}$ tends to $p_{a} / N$.

\section{Appendix A}

\section{Proof of Lemma 2.5}

We prove the inequality (49) by contradiction. Assume that there exists a sequence $\left(d_{n}^{0}, d_{n}^{1}\right)_{n}$ in $X_{D} \times L^{2}\left(\Omega ; H_{\#}^{1}\left(Y_{S}\right) / \mathbb{R}\right)^{N}$ such that $\left\|e_{x}\left(d_{n}^{0}\right)+e_{y}\left(d_{n}^{1}\right)\right\|_{L^{2}\left(\Omega \times Y_{S}\right)}$ tends to zero as $n$ goes to infinity and such that $\left\|d_{n}^{0}\right\|_{L^{2}(\Omega)}+\left\|d_{n}^{1}\right\|_{L^{2}\left(\Omega ; L_{\#}^{2}\left(Y_{S}\right) / \mathbb{R}\right)}+\left\|\nabla_{x} d_{n}^{0}\right\|_{L^{2}(\Omega)}+\left\|\nabla_{y} d_{n}^{1}\right\|_{L^{2}\left(\Omega \times Y_{S}\right)}=1$. Thus, up to 


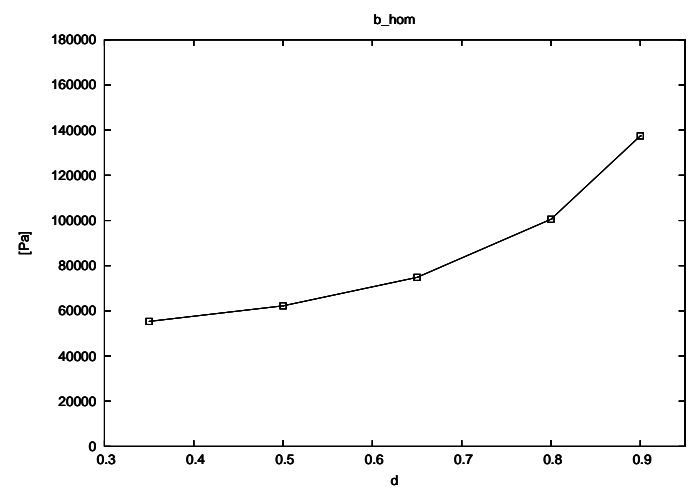

Figure 4: $b_{\text {hom }}=\frac{p_{a}|Y|}{N\left|Y_{S}\right|}$ for different diameters $d_{F}$.

a subsequence

$$
\begin{aligned}
& d_{n}^{0} \rightarrow d^{0} \text { in } L^{2}(\Omega)^{N}, \\
& d_{n}^{0} \rightarrow d^{0} \text { in } H^{1}(\Omega)^{N}, \\
& d_{n}^{1} \rightarrow d^{1} \text { in } L^{2}\left(\Omega ; H_{\#}^{1}\left(Y_{S}\right) / \mathbb{R}\right)^{N},
\end{aligned}
$$

from which we deduce that $e_{x}\left(d^{0}\right)+e_{y}\left(d^{1}\right)=0$ in $\mathcal{D}^{\prime}\left(\Omega \times Y_{S}\right)$. This implies that all the second derivatives of $d^{1}$ with respect to $y$ are equal to zero. Therefore $d^{1}=0$ in $L^{2}\left(\Omega ; H_{\#}^{1}\left(Y_{S}\right) / \mathbb{R}\right)^{N}$ since $d^{1}$ is periodic in $y$ and $Y_{S}$ is connected. Consequently, $e_{x}\left(d^{0}\right)=0$ which leads to $d^{0}=0$ in $\Omega$ since $d^{0}=0$ on $\Gamma_{D}$ (see $[8]$ ). We just proved that

$$
\begin{aligned}
& d_{n}^{0} \rightarrow 0 \text { in } L^{2}(\Omega)^{N}, \\
& d_{n}^{0} \rightarrow 0 \text { in } H^{1}(\Omega)^{N}, \\
& d_{n}^{1} \rightarrow 0 \text { in } L^{2}\left(\Omega ; H_{\#}^{1}\left(Y_{S}\right) / \mathbb{R}\right)^{N} .
\end{aligned}
$$

Now, in order to obtain a contradiction, we have to prove that the last two convergences are in fact strong ones. We have that

$$
\partial_{y_{i} y_{j}} d_{n}^{1} \rightarrow 0 \text { in } L^{2}\left(\Omega ; H^{-1}\left(Y_{S}\right)\right)^{N}, \forall(i, j) \in\{1,2,3\}
$$

that yields the strong convergence of $\nabla_{y} d_{n}^{1}$ towards 0 in $L^{2}\left(\Omega ; L^{2}\left(Y_{S}\right) / \mathbb{R}\right)^{N \times N}$. Thus there exist vectors $c_{n}^{i}$ in $L^{2}(\Omega)^{N}$ such that $\nabla_{y}\left(d_{n}^{1}\right)_{i}+c_{n}^{i}$ converges strongly towards zero in $L^{2}\left(\Omega \times Y_{S}\right)^{N}$. Since $\nabla_{y} d_{n}^{1}$ converges weakly in $L^{2}\left(\Omega \times Y_{S}\right)^{N \times N}$ to zero, we know that $c_{n}^{i}$ tends weakly in $L^{2}\left(\Omega \times Y_{S}\right)^{N}$ to zero. Next, we prove that this convergence is strong. Thanks to lemma 2. 10 of [3], for any vector $\theta$ in $L^{2}(\Omega)^{N}$ there exists $\psi \in L^{2}\left(\Omega ; H_{\#}^{1}\left(Y_{S}\right)\right)^{N}$ such that

$$
\begin{aligned}
& \operatorname{div}_{y}(\psi)=0 \\
& \psi=0 \text { on } \partial Y_{F}, \\
& \int_{Y_{S}} \psi(x, y) d y=\theta(x), \\
& \|\psi\|_{L^{2}\left(\Omega ; H_{\#}^{1}\left(Y_{S}\right)\right)} \leq C\|\theta\|_{L^{2}(\Omega)} .
\end{aligned}
$$

Consequently, considering $\left(\psi_{n}^{i}\right)$ associated with $\left(c_{n}^{i}\right)$, we remark that $\left(\psi_{n}^{i}\right)$ is bounded in $L^{2}\left(\Omega ; H_{\#}^{1}\left(Y_{S}\right)\right)^{N}$, and thanks to the strong convergence in $L^{2}\left(\Omega \times Y_{S}\right)^{N}$ of $\nabla_{y}\left(d_{n}^{1}\right)_{i}+c_{n}^{i}$ to zero, we have :

$$
\left\|c_{n}^{i}\right\|_{L^{2}(\Omega)}^{2}=\int_{\Omega} \int_{Y_{S}}\left(\nabla_{y}\left(d_{n}^{1}\right)_{i}+c_{n}^{i}\right) \cdot \psi_{n}^{i} \rightarrow 0
$$


Therefore

$$
d_{n}^{1} \rightarrow 0 \text { in } L^{2}\left(\Omega ; H_{\#}^{1}\left(Y_{S}\right) / \mathbb{R}\right)^{N}
$$

and

$$
e_{y}\left(d_{n}^{1}\right) \rightarrow 0 \text { in } L^{2}\left(\Omega \times Y_{S}\right)
$$

Thus

$$
e_{x}\left(d_{n}^{0}\right) \rightarrow 0 \text { in } L^{2}(\Omega)
$$

which implies thanks to Korn inequality on $X_{D}$ that $d_{n}^{0}$ converges strongly to zero in $X_{D}$. But then $\left\|d_{n}^{0}\right\|_{L^{2}(\Omega)}+\left\|d_{n}^{1}\right\|_{L^{2}\left(\Omega ; L_{\#}^{2}\left(Y_{S}\right) / \mathbb{R}\right)}+\left\|\nabla_{x} d_{n}^{0}\right\|_{L^{2}(\Omega)}+\left\|\nabla_{y} d_{n}^{1}\right\|_{L^{2}\left(\Omega \times Y_{S}\right)}=1 \rightarrow 0$, which leads to a contradiction.

\section{Appendix B}

\section{Proof of Proposition 2.6}

Proof. We will proceed as in [10]. Let $a_{y}^{\#}(\cdot, \cdot)$ be the bilinear form associated to problems $(L P)_{k l}$, that is

$$
a_{y}^{\#}(\chi, \varphi)=\frac{1}{\left|Y_{S}\right|}\left(\int_{Y_{S}} \mathcal{A} e_{y}(\chi): e_{y}(\varphi) d y+A \int_{\Gamma} \chi \cdot n d \gamma \int_{\Gamma} \varphi \cdot n d \gamma\right)
$$

We will prove that

$$
\mathcal{A}_{i j k l}^{h o m}=a_{y}^{\#}\left(\phi^{i j}, \phi^{k l}\right),
$$

where $\phi^{k l}=p^{k l}+\chi^{k l}$, and therefore part a) follow directy since it is easy to see that $a_{y}^{\#}(\cdot, \cdot)$ is a symmetric bilinear form. Part b) follows from part c) and the fact that $a_{y}^{\#}(\cdot, \cdot)$ is also positive.

In order to prove (88), we note that

$$
a_{y}^{\#}\left(\phi^{i j}, \phi^{k l}\right)=a_{y}^{\#}\left(\phi^{k l}, p^{i j}\right)+a_{y}^{\#}\left(\phi^{k l}, \chi^{i j}\right) .
$$

Let us study the first term in the right-hand side of (89):

$$
\begin{aligned}
a_{y}^{\#}\left(\phi^{k l}, p^{i j}\right)= & \frac{1}{\left|Y_{S}\right|} \int_{Y_{S}} \mathcal{A} e\left(\phi^{k l}\right): e\left(p^{i j}\right) d y+\frac{A}{\left|Y_{S}\right|} \int_{\Gamma} \phi^{k l} \cdot n d \gamma \int_{\Gamma} p^{i j} \cdot n d \gamma \\
= & \frac{1}{\left|Y_{S}\right|} \int_{Y_{S}} \sigma\left(\phi^{k l}\right)_{i j} d y \\
& +\frac{A}{\left|Y_{S}\right|}\left(\int_{Y_{S}} \operatorname{div} \chi^{k l} d y-\int_{Y_{F}} \operatorname{div} p^{k l} d y\right)\left(-\int_{Y_{F}} \operatorname{div} p^{i j} d y\right) \\
= & \frac{1}{\left|Y_{S}\right|}\left(\int_{Y_{S}} \sigma\left(\phi^{k l}\right)_{i j} d y-A\left|Y_{F}\right| \delta_{i j} \int_{Y_{S}} \operatorname{div} \chi^{k l} d y+A\left|Y_{F}\right|^{2} \delta_{k l} \delta_{i j}\right),
\end{aligned}
$$

where we have used that $\operatorname{div} p^{k l}=\delta_{k l}$ and $e\left(p^{k l}\right)_{i j}=\delta_{i j k l}$. Hence, $a_{y}^{\#}\left(\phi^{k l}, p^{i j}\right)=\mathcal{A}_{i j k l}^{\text {hom }}$.

Next, we will prove that the second term in the right-hand side of (89) is equal to 0. In fact, we use $\chi^{i j}$ as test function in $(L P)_{k l}$, then, after an integration by parts and using the boundary condition on $\Gamma$, we obtain

$$
\left|Y_{S}\right| a_{y}^{\#}\left(\chi^{k l}, \chi^{i j}\right)+\int_{\Gamma} \sigma^{1}\left(p^{k l}\right) n \cdot \chi^{i j} d \gamma=0 .
$$


Integration by parts of the second term in the left-hand side of (90) gives

$$
\int_{\Gamma} \sigma^{1}\left(p^{k l}\right) n \cdot \chi^{i j} d \gamma=\int_{Y_{S}} \operatorname{div}_{y} \sigma^{1}\left(p^{k l}\right) \chi^{i j} d y+\int_{Y_{S}} \sigma^{1}\left(p^{k l}\right): e\left(\chi^{i j}\right) d y,
$$

and since $p^{k l}$ is a degree one polynomial we have

$$
\int_{\Gamma} \sigma^{1}\left(p^{k l}\right) n \cdot \chi^{i j} d \gamma=\int_{Y_{S}} \sigma^{1}\left(p^{k l}\right): e\left(\chi^{i j}\right) d y .
$$

Combining with (90) we obtain

$$
\left|Y_{S}\right| a_{y}^{\#}\left(\chi^{k l}, \chi^{i j}\right)=-\int_{Y_{S}} \sigma^{1}\left(p^{k l}\right): e\left(\chi^{i j}\right) d y .
$$

On the other hand,

$$
\begin{aligned}
a_{y}^{\#}\left(p^{k l}, \chi^{i j}\right) & =\frac{1}{\left|Y_{S}\right|}\left(\int_{Y_{S}} \sigma\left(p^{k l}\right): e\left(\chi^{i j}\right) d y+A \int_{\Gamma} p^{k l} \cdot n d \gamma \int_{\Gamma} \chi^{i j} \cdot n d \gamma\right) \\
& =\frac{1}{\left|Y_{S}\right|}\left(\int_{Y_{S}} \sigma\left(p^{k l}\right): e\left(\chi^{i j}\right) d y-A\left|Y_{F}\right| \int_{Y_{S}} \operatorname{div}_{y} \chi^{i j} d y\right) \\
& =\frac{1}{\left|Y_{S}\right|} \int_{Y_{S}} \sigma^{1}\left(p^{k l}\right): e\left(\chi^{i j}\right) d y .
\end{aligned}
$$

Therefore, by adding (91) and (92) we conclude that $a_{y}^{\#}\left(\phi^{k l}, \chi^{i j}\right)=0$.

Part c) is consequence of (88). In fact

$$
\begin{aligned}
0=\mathcal{A}^{\text {hom }} \xi: \xi & =\sum_{i j} \sum_{k l} \mathcal{A}_{i j k l}^{h o m} \xi_{i j} \xi_{k l} \\
& =a_{y}^{\#}\left(\sum_{i j} \xi_{i j} \phi^{i j}, \sum_{k l} \xi_{k l} \phi^{k l}\right) \\
& =a_{y}^{\#}\left(\phi_{\xi}, \phi_{\xi}\right),
\end{aligned}
$$

where $\phi_{\xi}=\sum_{i j} \xi_{i j} \phi^{i j}$ and $\sum_{i j}(\cdot)=\sum_{1 \leq i, j \leq N}(\cdot)$. (93) implies that $e_{y}\left(\phi_{\xi}\right)=0$ and then (see [22]), if $N=3$, we have that

$$
\phi_{\xi}=a+b \times y,
$$

with $a, b \in \mathbb{R}^{3}$. That is, $\phi_{\xi}$ is a rigid displacement of a solid body (see [31]). On the other hand

$$
\phi_{\xi}=\sum_{i j} \xi_{i j} \phi^{i j}=\sum_{i j} \xi_{i j}\left(\chi^{i j}+p^{i j}\right) .
$$

Combining (94) and (95) we have that $\sum_{i j} \xi_{i j} \chi^{i j}$ is a polynomial of degree one. Since $\chi^{i j}$ is $Y$-periodic we conclude that

$$
\sum_{i j} \xi_{i j} \chi^{i j}=c, c \in \mathbb{R}^{N}
$$


Therefore

$$
\phi_{\xi}=c+\sum_{i j} \xi_{i j} p^{i j}
$$

Combining now (94) and (96) we have

$$
a+b \times y=c+\sum_{i j} \xi_{i j} p^{i j}, \forall y \in \mathbb{R}^{3},
$$

in particular, for $y=0$, then using that $p^{i j}(0)=0$, we have $a=c$ and then

$$
b \times y=\xi y
$$

which implies that $b=0$ and $\xi=0$. When $N=2$ a similar analysis can be performed.

\section{Appendix C}

\section{Proof of Lemma 3.6}

Proof. Lemma 3.6 is equivalent to show that for any $p \in L^{2}\left(\Omega ; L_{\#}^{2}\left(Y_{S}\right)\right)$ there exists $\left(\phi, \phi^{1}\right) \in$ $X_{D} \times L^{2}\left(\Omega ; H_{\#}^{1}\left(Y_{S}\right) / \mathbb{R}\right)^{N}$ such that

$$
\operatorname{div}_{x} \phi+\operatorname{div}_{y} \phi^{1}=p, \text { in } \Omega \times Y_{S}
$$

and

$$
\left\|\left(\phi, \phi^{1}\right)\right\|_{X_{D} \times L^{2}\left(\Omega, H_{\#}^{1}\left(Y_{S}\right) / \mathbb{R}\right)^{N}} \leq C\|p\|_{L^{2}\left(\Omega ; L_{\#}^{2}\left(Y_{S}\right)\right)} .
$$

We built $\bar{p}$ such that

$$
\bar{p}= \begin{cases}p, & \text { in } \Omega \times Y_{S}, \\ -\frac{1}{\left|Y_{S}\right|} \int_{\Omega} \int_{Y_{S}} p(x, y) d x d y, & \text { in } \Omega \times Y_{F} .\end{cases}
$$

The new pressure $\bar{p}$ satisfies $\int_{\Omega} \int_{Y} \bar{p}(x, y) d x d y=0$. We would like to find $\left(\phi, \phi^{1}\right) \in X_{D} \times$ $L^{2}\left(\Omega ; H_{\#}^{1}(Y) / \mathbb{R}\right)^{N}$ such that $\operatorname{div}_{x} \phi+\operatorname{div}_{y} \phi^{1}=p$ in $\Omega \times Y$. If such $\left(\phi, \phi^{1}\right)$ exists then it verifies

$$
\operatorname{div}_{x} \phi=\frac{1}{|Y|} \int_{Y} \bar{p}=\chi \in L_{0}^{2}(\Omega)
$$

and

$$
\operatorname{div}_{y} \phi^{1}=\bar{p}-\frac{1}{|Y|} \int_{Y} \bar{p}=\theta
$$

where $\theta \in L^{2}\left(\Omega ; L_{\#}^{2}(Y)\right)$ and $\int_{Y} \theta=0$. We know (see [16], [34]) that there exists $\phi \in H_{0}^{1}(\Omega)^{N}$ such that $\operatorname{div}_{x} \phi=\chi$ and $\|\phi\|_{H_{0}^{1}(\Omega)} \leq C\|\chi\|_{L_{0}^{2}(\Omega)}$. But $\|\chi\|_{L_{0}^{2}(\Omega)} \leq C\|p\|_{L^{2}\left(\Omega ; L_{\#}^{2}\left(Y_{S}\right)\right)}$ thus $\|\phi\|_{H_{0}^{1}(\Omega)} \leq$ $C\|p\|_{L^{2}\left(\Omega ; L_{\#}^{2}\left(Y_{S}\right)\right)}$. In order to find $\phi^{1}$ we solve

$$
\left\{\begin{array}{l}
\Delta_{y} \chi^{1}=\theta, \quad \text { in } Y, \\
\frac{\partial \chi^{1}}{\partial n}=0, \quad \text { on } \Gamma \\
\chi^{1} \text { periodic in } y .
\end{array}\right.
$$


This problem is well-posed since $\int_{Y} \theta=0$, and we have

$$
\left\|\chi^{1}(x, \cdot)\right\|_{H^{1}(Y) / \mathbb{R}} \leq C\|\theta(x, \cdot)\|_{L^{2}(Y)} .
$$

Consequently, since $\|\theta\|_{L^{2}\left(\Omega ; L_{\#}^{2}(Y)\right)} \leq C\|p\|_{L^{2}\left(\Omega ; L_{\#}^{2}\left(Y_{S}\right)\right)}, \phi^{1}=\nabla_{y} \chi^{1}$ answers the question.

Acknowledgements. The last author acknowledges the support of the CNRS for a three month stay at the Jacques-Louis Lions Laboratory, University Pierre et Marie Curie - Paris 6, France in 2005 , since a part of this joint work was possible thanks to this stay. The first author acknowledges the support of the following institutions, where part of this work has been developed: JacquesLouis Lions Laboratory, University Paris 6, France, through ACI project "le-poumon-vous-dis-je"; CMM, University of Chile, Santiago-Chile, through CONICYT-FONDAP project; CEREMADE, University of Paris-Dauphine, France (for supporting a one month stay at CEREMADE in 2005); and Department of Mathematical Engineering, University of Concepción, Concepción-Chile.

\section{References}

[1] Y. Achdou, C. Sabot, N. Tchou, Diffusion and propagation in some ramified domains with a fractal boundary, M2AN Math. Model. Numer. Anal. 40 (2006), no. 4, 623-652.

[2] Y. Achdou, C. Sabot, N. Tchou, A multiscale method for some Poisson problems in ramifed domains with fractal boundaries, Multiscale Model. Simul. 5 (2006), no. 3, 828860.

[3] G. Allaire, Homogenization and two-scale convergence. SIAM J. Math. Anal. 23 (1992), no. $6,1482-1518$.

[4] G. Allaire, Homogenization of the unsteady Stokes equation in porous media. Pitman Res. Notes Math. Ser., 267, Longman Sci. Tech., Harlow (1992).

[5] G. Allaire, C. Conca, Bloch-wave homogenization for a spectral problem in fluid-solid structures. Arch. Rational Mech. Anal. 135 (1996), 197-257.

[6] A. Bensoussan, J.-L. Lions, G. Papanicolaou, Asymptotic analysis for periodic structures, Studies in Mathematics and its Applications, Vol. 5, North-Holland, Amsterdam, 1978.

[7] M. Briane, Y. Maday, F. Madigou, Homogenization of a two-dimensional fractal conductivity, in preparation.

[8] P. G. Ciarlet, Mathematical Elasticity. Vol. 1, Studies in Mathematics and its Applications (20), North-Holland, 1987.

[9] D. Cioranescu, A. Piatnitski, Homogenization in perforated domains with rapidly pulsing perforations, ESAIM Control Optim. Calc. Var., 9 (2003), 461-483.

[10] C. Conca, On the application of the homogenization theory to a class of problems arising in fluid mechanics, J. Math. pures et appl,. 64 (1985), 31-75.

[11] C. Conca, Numerical results on the homogenization of Stokes and Navier-Stokes equations modeling a class of problems from fluid mechanics, Comput. Methods Appl. Mech. Engrg. 53 (1985), 223-258. 
[12] C. Conca, J. Planchard, M. Vanninathan, Fluids and Periodic Structures, Collection RMA, Masson, Paris, (1995).

[13] G. Duvaut, J.-L. Lions, Les inéquations en mécanique et en physique, Travaux et Recherches Mathématiques, 21, Dunod, Paris, 1972.

[14] I. A. Ene, J. Saint Jean Paulin, Homogenization and two scale convergence for a Stokes or Navier-Stokes flow in an elastic thin porous medium. M3AS 6 (1996), no. 7, 941-945.

[15] C. Fetita, S. Mancini, D. Perchet, F. Prêteux, M. Thiriet, L. Vial, An image based computational model of oscillatory flow in the proximal part of tracheobronchial trees. Comput. Meth. Biomech. Biomed. Eng. 8 (2005), no. 4, 279-293.

[16] V. Girault, P.-A. Raviart, Finite Element Method for Navier-Stokes Equations. Theory and Algorithms, Springer Series in Computational Mathematics, 5, Springer-Verlag, Berlin, 1986.

[17] C. Grandmont, Y. Maday, B. Maury, A multiscale/multimodel approach of the respiration tree. New trends in continuum mechanics, Theta Ser. Adv. Math., 3, Theta, Bucharest, (2005), 147-157.

[18] C. Grandmont, B. Maury, N. Meunier, A viscoelastic model with non-local damping application to the human lungs. M2AN Math. Model. Numer. Anal. 40 (2006), no. 1, 201-224.

[19] H. Gray, Anatomy of the Human Body, Philadelphia: Lea \& Febiger, 1918; Bartleby.com, 2000. www. bartleby.com/107/.

[20] Q. Grimal, Etude dans le domaine temporel de la propagation d'ondes élastiques en milieux stratifiés; Modélisation de la réponse du thorax à un impact, Thesis Université de Paris XIII, 2003.

[21] Q. Grimal, A. Watzky, S. Nali, A one-dimensional model for the propagation of pressure waves through the lung. Journal of Biomechanics, 35 (2002), 1081-1089.

[22] I. Hlaváček, J. Nečas, On Inequalities of Korn's Type, II. Applications to Linear Elasticity. Arch. Rational Mech. Anal. 36 (1970), 312-334.

[23] H. Kitaoka, S. Tamura, R. Takaki, A three-dimensional model of the human pulmonary acinus, J. Appl. Physiol. 88 (2000), 2260-2268.

[24] A. E. Løvgren, Y. Maday, E. M. Rønquist, A reduced basis element method for complex fluid systems, in Proceedings European Conference on Computational Fluid Dynamics, ECCOMAS CFD 2006, P. Wesseling, E. Oñate, J. Periaux (Editors), 2006.

[25] B. Mauroy, M. Filoche, J. S. Andrade Jr., B. Sapoval, Interplay between geometry and flow distribution in an airway tree, Phys. Rev. Let., 2003, 90, 148101 1-4.

[26] B. Mauroy, M. Filoche, E. R. Weibel Jr., B. Sapoval, An optimal bronchial tree may be dangerous, Nature, 2004, 427, 633-636.

[27] B. Maury, N. Meunier, A. Soualah, L. Vial, Outlet dissipative conditions for air flow in the bronchial tree, ESAIM Proceedings, 14 (2005), 201-212. 
[28] G. Nguetseng, A general convergence result for a functional related to the theory of homogenization, SIAM J. Math. Anal., 20 (1989), 608-623.

[29] M. Owen, M. Lewis, The Mechanics of Lung Tissue under High-Frequency Ventilation. SIAM J. Appl. Math., 61 (2001), no. 5, 1731-1761.

[30] A. Quarteroni, A. Veneziani, Analysis of a geometrical multiscale model based on the coupling of ODEs and PDEs for blood flow simulations, SIAM Multiscale Model. Simul. 1 (2003), no. 2, 173-195.

[31] P.-A. Raviart, J.M. Thomas, Introduction à l'analyse numérique des équations en dérivées partielles, 2nd édition, Masson, Paris, 1988.

[32] E. Sanchez-Palencia, Nonhomogeneous media and vibration theory, Lecture Notes in Phys., Vol. 127, Springer-Verlag, Berlin, 1980.

[33] L. Tartar, Incompressible fluid flow in a porous medium. Convergence of the homogenization process. Appendix in E. Sanchez-Palencia, Nonhomogeneous media and vibration theory, Lecture Notes in Phys., Vol. 127, Springer-Verlag, Berlin, 1980.

[34] R. Temam, Navier-Stokes equations. Theory and numerical analysis. Third edition. Studies in Mathematics and its Applications, 2. North-Holland Publishing Co., Amsterdam, 1984

[35] R. S. Tepper, B. Wiggs, S. J. Gunst, P. D. Paré, Comparison of the shear modulus of mature and immature rabbit lungs, J. Appl. Physiol. 87 (1999), 711-714.

[36] E. R. Weibel, Morphometry of the human lung, Springer Verlag, 1963.

[37] E. R. Weibel, The pathway for oxygen, Harvard University Press, December 1984. 